\title{
RELAP-7 Numerical Stabilization: Entropy Viscosity Method
}

R. A. Berry

M. O. Delchini

J. Ragusa

June 2014

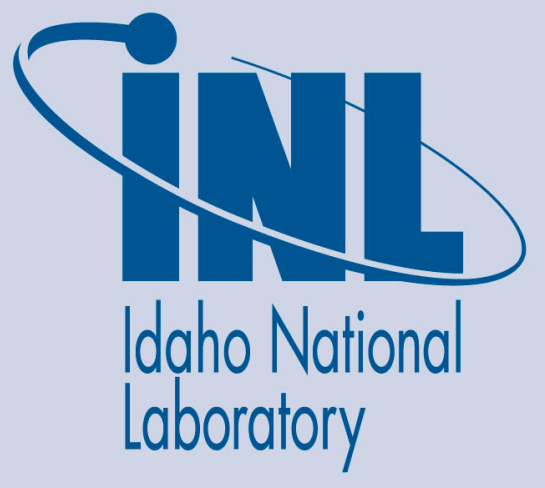

The INL is a U.S. Department of Energy National Laboratory operated by Battelle Energy Alliance 
INL/EXT-14-32352

\title{
RELAP-7 Numerical Stabilization: Entropy Viscosity Method
}

${ }^{1}$ Texas A\&M University

\author{
R. A. Berry \\ M. O. Delchini ${ }^{1}$ \\ J. Ragusa ${ }^{1}$
}

June 2014

\section{Idaho National Laboratory \\ Idaho Falls, Idaho 83415}

http://www.inl.gov

\author{
Prepared for the \\ U.S. Department of Energy \\ Office of Nuclear Energy \\ Under DOE Idaho Operations Office \\ Contract DE-AC07-05ID14517
}




\section{INL REPORT}

INL/EXT-14-32352

Unlimited Release

Printed June 2014

\section{RELAP-7 Numerical Stabilization: Entropy Viscosity Method}

Prepared by

Idaho National Laboratory

Idaho Falls, Idaho 83415

The Idaho National Laboratory is a multiprogram laboratory operated by Battelle Energy Alliance for the United States Department of Energy under DOE Idaho Operations Office. Contract DE-AC07-05ID14517.

Approved for public release; further dissemination unlimited.

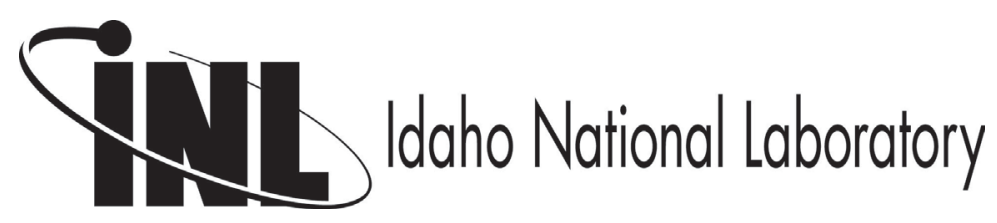


Issued by the Idaho National Laboratory, operated for the United States Department of Energy by Battelle Energy Alliance.

NOTICE: This report was prepared as an account of work sponsored by an agency of the United States Government. Neither the United States Government, nor any agency thereof, nor any of their employees, nor any of their contractors, subcontractors, or their employees, make any warranty, express or implied, or assume any legal liability or responsibility for the accuracy, completeness, or usefulness of any information, apparatus, product, or process disclosed, or represent that its use would not infringe privately owned rights. Reference herein to any specific commercial product, process, or service by trade name, trademark, manufacturer, or otherwise, does not necessarily constitute or imply its endorsement, recommendation, or favoring by the United States Government, any agency thereof, or any of their contractors or subcontractors. The views and opinions expressed herein do not necessarily state or reflect those of the United States Government, any agency thereof, or any of their contractors.

Printed in the United States of America. This report has been reproduced directly from the best available copy.

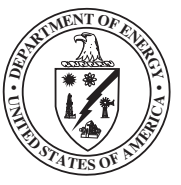


INL/EXT-14-32352

Unlimited Release

Printed June 2014

\title{
RELAP-7 Numerical Stabilization: Entropy Viscosity Method
}

\author{
R. A. Berry, M. O. Delchini * , J. Ragusa ${ }^{\dagger}$
}

\footnotetext{
${ }^{*}$ Texas A\&M University, PhD Candidate

${ }^{\dagger}$ Texas A\&M University, Associate Professor
} 


\section{Contents}

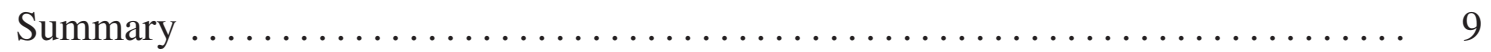

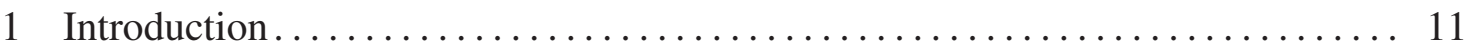

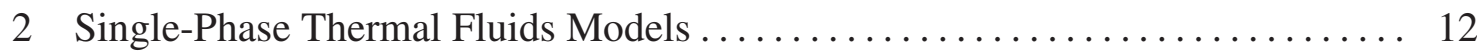

2.1 Single-Phase Flow Model . . . . . . . . . . . . . . . . . . . 12

2.1.1 Single-Phase Flow Field Equations . . . . . . . . . . . . . . . . . . 12

2.2 Single-Phase Flow Constitutive Models . . . . . . . . . . . . . . 12

2.2 .1 Equations of State . . . . . . . . . . . . . . . 12

2.2.2 Stiffened Gas Equation of State . . . . . . . . . . . . . 13

2.2 .3 Ideal Gas Equation of State . . . . . . . . . . . . . . . 15

3 Two-Phase Thermal Fluids Models ............................. 16

3.1 Seven Equation Two-Phase Flow Model . . . . . . . . . . . . . . . . 16

3.1.1 One-dimensional, Variable Cross-sectional Area, Seven Equation

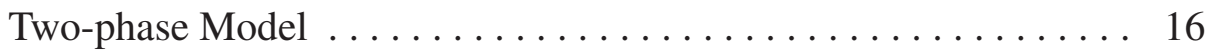

3.2 Seven-Equation Two-Phase Flow Constitutive Models . . . . . . . . . . . . . 18

3.2.1 Interface Pressure and Velocity, Mechanical Relaxation Coefficients 19

3.2 .2 Wall and Interphase Friction . . . . . . . . . . . . . 20

3.2.3 Wall and Interface Direct Heat Transfer . . . . . . . . . . . . . . 21

3.2.4 Interphase Mass Transfer . . . . . . . . . . . . . . . . 21

3.2.5 Stiffened Gas Equation of State for Two-phase Flows . . . . . . . . . 25

4 Numerical Methods and Entropy Viscosity Stabilization .................. 28

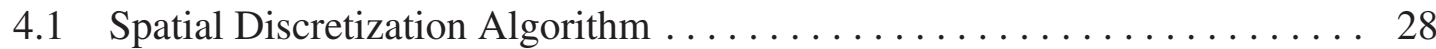

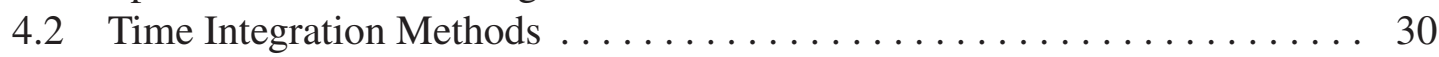

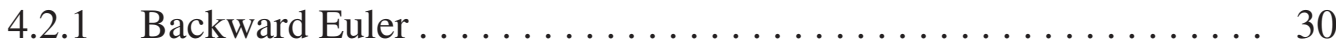

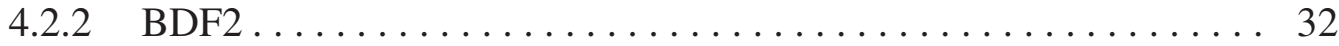

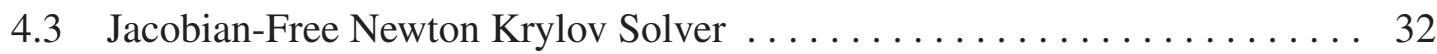

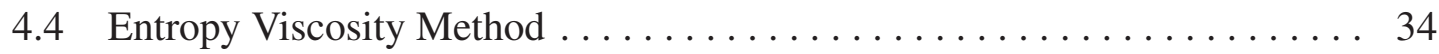

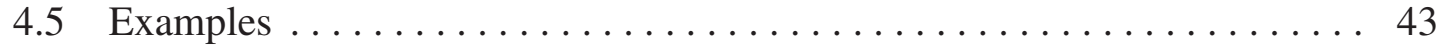

4.5.1 Constant wall heat source and wall friction $\ldots \ldots \ldots \ldots \ldots . \ldots 44$

4.5.2 Constant wall temperature and wall friction . . . . . . . . . . 45

4.5.3 Mass and heat exchange terms, and wall friction . . . . . . . . . 46

4.5.4 1-D converging-diverging duct with relaxation terms . . . . . . . 46

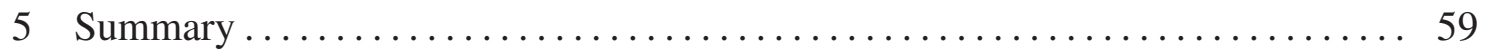

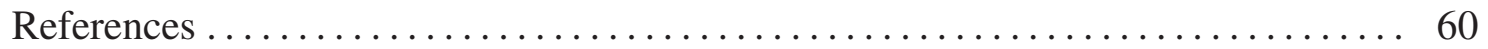




\section{Figures}

1 Interface control volume (top); $T-p$ state space around saturation line,

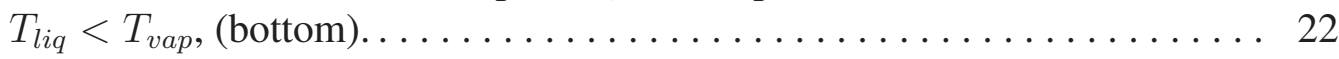

2 Vaporization and condensation at a liquid-vapor interface (after Moody [1]). 24

3 Steady-state pressure profile with constant wall-heat flux and mass flow rate boundary............................. 47

4 Steady-state temperature profile with constant wall-heat flux and mass flow rate boundary. ........................... 47

5 Steady-state velocity profile with constant wall-heat flux and mass flow rate boundary........................... 48

6 Steady-state viscosity coefficients profile with constant wall-heat flux and mass flow rate boundary. ......................... 48

7 Steady-state pressure profile with constant wall-heat flux and stagnation boundary. ................................. 49

8 Steady-state temperature profile with constant wall-heat flux and stagnation boundary. ............................... 50

9 Steady-state velocity profile with constant wall-heat flux and stagnation boundary. ................................ 51

10 Steady-state viscosity coefficients profile with constant wall-heat flux and stagnation boundary. .............................. 51

11 Steady-state temperature profile with constant wall-heat transfer and first-

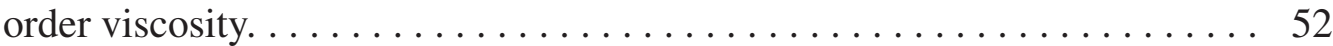

12 Steady-state pressure profile with constant wall temperature and mass flow

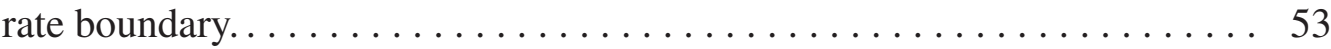

13 Steady-state temperature profile with constant wall temperature and mass

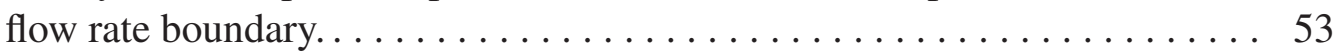

14 Steady-state velocity profile with constant wall temperature and mass flow rate boundary..................................... 54

15 Steady-state viscosity coefficients profile with constant wall temperature and mass flow rate boundary. ...................... 54

16 Steady-state pressure profile with friction and relaxation terms. . . . . . . 55

17 Steady-state velocity profile with friction and relaxation terms. ........ 56

18 Steady-state viscosity coefficients profile with friction and relaxation terms. 56

19 Steady-state pressure profiles in a converging-diverging duct with pressure and velocity relaxation terms. ..................... 57

20 Steady-state velocity profiles in a converging-diverging duct with pressure and velocity relaxation terms. ........................ 57 
21 Steady-state volume fraction profiles in a converging-diverging duct with

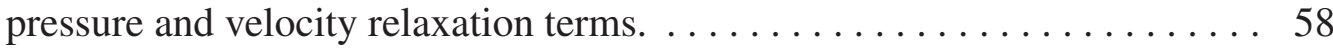

22 Steady-state viscosity coefficient profiles in a converging-diverging duct with pressure and velocity relaxation terms. . . . . . . . . . 58 


\section{Tables}

1 Stiffened gas equation of state parameters for water and its vapor, from [2]. 14

2 Boundary conditions. ....................... 44 


\section{Summary}

The RELAP-7 code is the next generation nuclear reactor system safety analysis code being developed at the Idaho National Laboratory (INL). The code is based on the INL's modern scientific software development framework, MOOSE (Multi-Physics Object Oriented Simulation Environment). The overall design goal of RELAP-7 is to take advantage of the previous thirty years of advancements in computer architecture, software design, numerical integration methods, and physical models. The end result will be a reactor systems analysis capability that retains and improves upon RELAP5's capability and extends the analysis capability for all reactor system simulation scenarios.

RELAP-7 utilizes a single phase and a novel seven-equation two-phase flow models as described in the RELAP-7 Theory Manual [3]. The basic equation systems are hyperbolic, which generally require some type of stabilization (or artificial viscosity) to capture nonlinear discontinuities and to suppress advection-caused oscillations. This report documents one of the available options for this stabilization in RELAP-7 - a new and novel approach known as the entropy viscosity method.

Because the code is an ongoing development effort in which the physical sub models, numerics, and coding are evolving, so too must the specific details of the entropy viscosity stabilization method. Here the fundamentals of the method in their current state are presented. 


\section{Introduction}

The RELAP-7 (Reactor Excursion and Leak Analysis Program) code [3] is the next generation nuclear reactor system safety analysis code being developed at Idaho National Laboratory (INL). The primary basis of the RELAP-7 governing theory includes 7-equation two-phase flow, reactor core heat transfer, and reactor kinetics models. Based on the INL's modern scientific software development framework MOOSE (Multi-Physics Object Oriented Simulation Environment) [4], RELAP-7 endeavors to take advantage of the previous thirty years of advancements in computer architecture, software design, numerical integration methods, and physical models.

The basic equation systems for the flow, both single- and two-phase are hyperbolic. Hyperbolic equation systems generally require some type of stabilization (or artificial viscosity) to capture nonlinear discontinuities and to suppress advection-caused oscillations. RELAP-7 has multiple methods available for stabilization which are well documented in the literature. However, one of the methods for stabilization available within RELAP-7 is a new and novel approach, called the entropy viscosity method, which is not yet well documented in the literature. This stabilization method is based on the independent doctoral research of M. O. Delchini, under INL sponsorship, along with colleagues, at Texas A \& M University and INL.

The purpose of this report is to present the fundamental basis for the entropy viscosity stabilization method. Because it is to be applied to both a single phase model as well as a very complex two-phase model, for completeness a summary of the RELAP-7 flow models and numerical methods is given. For more details, the reader is referred to [3]. 


\section{Single-Phase Thermal Fluids Models}

\subsection{Single-Phase Flow Model}

RELAP-7 treats the basic pipe, duct, or channel flow component as being one dimensional with a cross-sectional area that varies along its length.

\subsubsection{Single-Phase Flow Field Equations}

The single-phase specific area averaged mass, momentum, total energy, and entropy balances can be respectively written as

$$
\begin{aligned}
\frac{\partial \rho A}{\partial t}+\frac{\partial \rho u A}{\partial x} & =0 \\
\frac{\partial \rho u A}{\partial t}+\frac{\partial\left(\rho u^{2} A+p A\right)}{\partial x} & =\tilde{p} \frac{\partial A}{\partial x}-F_{\text {wall friction }} \\
\frac{\partial \rho E A}{\partial t}+\frac{\partial(\rho E+p) u A}{\partial x} & =-\tilde{p} \frac{\partial A}{\partial t}-Q_{\text {wall }} \\
\frac{\partial \rho s A}{\partial t}+\frac{\partial \rho s u A}{\partial x}+\frac{\partial}{\partial x}\left(\frac{q_{x} A}{T}\right)-A \Delta & =-\frac{Q_{\text {wall }}}{\tilde{T}}
\end{aligned}
$$

where the $F_{\text {wall friction }}$ is the average duct wall shear force (friction), $Q_{\text {wall }}$ is the average heat flux from the fluid to the duct wall and $\tilde{T}$ is the average fluid temperature along the line $c$ on the duct wall.

\subsection{Single-Phase Flow Constitutive Models}

\subsubsection{Equations of State}

To close the thermodynamical portion of the balance equation systems of RELAP-7 an equation of state must be specified to reflect the material specific relationships among the thermodynamic variables. It suites our purpose here to define a pair of equations

$$
\begin{aligned}
p & =p(\rho, e) \\
T & =T(\rho, e)
\end{aligned}
$$


i.e., both the pressure and the temperature can be computed if the density and internal energy are given. Reformulations of (5) and (6) which consist of two equations relating the four quantities $p, T, \rho$, and $e$ are also acceptable and useful in practice.

More will be said subsequently, when discussing selection and stabilization of "weak" solutions.

\subsubsection{Stiffened Gas Equation of State}

In the single-phase model discussed in this section, the fluid (whether it be liquid or vapor) is compressible and behaves with its own convex equation of state (EOS). For initial development purposes it was decided to use a simple form capable of capturing the essential physics. For this purpose, the stiffened gas equation of state (SGEOS) was selected (LeMetayer et al. [2])

$$
p(\rho, e)=(\gamma-1) \rho(e-q)-\gamma p_{\infty}
$$

where $p, \rho, e$, and $q$ are the pressure, density, internal energy, and the binding energy of the fluid considered. The parameters $\gamma, q$, and $p_{\infty}$ are the constants (coefficients) of each fluid. The parameter $q$ defines the zero point for the internal energy, which will be relevant later when phase transitions are involved with two-phase flows. The parameter $p_{\infty}$ gives the "stiffened" properties compared to ideal gases, with a large value implying "nearly-incompressible" behavior.

The first term on the right-hand side of (7) is a repulsive effect that is present for any state (gas, liquid, or solid), and is due to molecular motions and vibrations. The second term on the right represents the attractive molecular effect that guarantees the cohesion of matter in the liquid or solid phases. The parameters used in this equation of state are determined by using a reference curve, usually in the $\left(p, \frac{1}{\rho}\right)$ plane. In LeMetayer et al. [2], the saturation curves are utilized as this reference curve to determine the stiffened gas parameters for liquid and vapor phases. The SGEOS is the simplest prototype that contains the main physical properties of pure fluids - repulsive and attractive molecular effects - thereby facilitating the handling of the essential physics and thermodynamics with a simple analytical formulation. Thus, a fluid, whether liquid or vapor, has its own thermodynamics.

The pressure law, equation (7), is incomplete. A caloric law is also needed to relate the fluid temperature to the other fluid properties (for example, $T=T(p, \rho)$ ) and thereby completely describe the thermodynamic state of the fluid. For the fluid, whether liquid or 
vapor, it is assumed that the thermodynamic state is determined by the SGEOS as:

$$
\begin{aligned}
e(p, \rho) & =\frac{p+\gamma p_{\infty}}{(\gamma-1) \rho}+q \\
\rho(p, T) & =\frac{p+p_{\infty}}{(\gamma-1) c_{v} T} \\
h(T) & =\gamma c_{v} T+q \\
g(p, T) & =\left(\gamma c_{v}-q^{\prime}\right) T-c_{v} T \ln \frac{T^{\gamma}}{\left(p+p_{\infty}\right)^{(\gamma-1)}}+q
\end{aligned}
$$

where $T, h$, and $g$ are the temperature, enthalpy, and Gibbs free enthalpy, respectively, of the fluid considered. In this system, equation (9) is the caloric law. In addition to the three material constants mentioned above, two additional material constants have been introduced, the constant volume specific heat $c_{v}$ and the parameter $q^{\prime}$. These parameters will be useful when two-phase flows are considered later. The values for water and its vapor from [2] are given in Table 1. These parameter values appear to yield reasonable approximations over a temperature range from 298 to $473 \mathrm{~K}$ [2]. Equation (10) can also

Table 1. Stiffened gas equation of state parameters for water and its vapor, from [2].

\begin{tabular}{cccccc}
\hline Water & $\gamma$ & $q\left(\mathrm{~J} \mathrm{~kg}^{-1}\right)$ & $q^{\prime}\left(\mathrm{J} \mathrm{kg}^{-1} \mathrm{~K}^{-1}\right)$ & $p_{\infty}(\mathrm{Pa})$ & $c_{v}\left(\mathrm{~J} \mathrm{~kg}^{-1} \mathrm{~K}^{-1}\right)$ \\
\hline Liquid & 2.35 & $-1167 \times 10^{3}$ & 0 & $10^{9}$ & 1816 \\
Vapor & 1.43 & $2030 \times 10^{3}$ & $-23 \times 10^{3}$ & 0 & 1040 \\
\hline
\end{tabular}

be written as

$$
h=c_{p} T+q
$$

if we define $c_{p}=\gamma c_{v}$. Combining (8) and (9) also allows us to write the temperature as

$$
T=\frac{1}{c_{v}}\left(e-q-\frac{p_{\infty}}{\rho}\right) .
$$

The sound speed for this equation of state can be computed as

$$
\begin{aligned}
c^{2} & =\frac{p}{\rho^{2}}(\gamma-1) \rho+(\gamma-1)(e-q) \\
& =\gamma\left(\frac{p+p_{\infty}}{\rho}\right) .
\end{aligned}
$$




\subsubsection{Ideal Gas Equation of State}

The ideal gas equation of state is fundamental; many other equations of state are more-orless based on the ideal gas equation of state in some way. Although RELAP-7 is primarily concerned with flows involving liquids and their vapors, there are certainly nuclear reactor applications, such as helium cooling, where the ideal gas equation of state is relevant. The pressure and temperature in a (calorically-perfect) ideal gas are given by

$$
\begin{aligned}
p & =(\gamma-1) \rho e \\
T & =\frac{e}{c_{v}}
\end{aligned}
$$

where $\gamma=\frac{c_{p}}{c_{v}}$ is the ratio of specific heats, and $c_{v}$ is the specific heat at constant volume, which in a calorically-perfect gas is assumed to be constant. This equation of state is a particular form of the stiffened gas equation of state described above, with $q=p_{\infty}=0$. 


\section{Two-Phase Thermal Fluids Models}

\subsection{Seven Equation Two-Phase Flow Model}

\subsubsection{One-dimensional, Variable Cross-sectional Area, Seven Equation Two-phase Model}

Because it is not economical to solve the entire two-phase flow field with highly resolved three-dimensional computational fluid dynamics for an entire light water reactor coolant system, it is necessary to construct a one-dimensional model for flow in pipes, nozzles, and other components. The one-dimensional model is constructed to allow the representation of continuously variable cross-sectional area.

Consider flow through a duct with local cross-sectional area $A=A(x, t)$. Actually, most of the time we consider local cross-sectional area to depend upon position coordinate $x$ only, for which a time rate of change of cross-sectional area is not necessary because for this case $\frac{\partial A}{\partial t}=0$. However, $A(x, t)$ is left inside the time derivative terms for generality and possible future use. The seven-equation two-phase system model can be stated as balances of mass, momentum, and total energy, along with volume fraction evolution as

$$
\begin{aligned}
\frac{\partial(\alpha \rho)_{l i q} A}{\partial t}+\frac{\partial(\alpha \rho u)_{l i q} A}{\partial x} & =-\Gamma A_{i n t} A \\
\frac{\partial(\alpha \rho u)_{l i q} A}{\partial t}+\frac{\partial \alpha_{l i q} A\left(\rho u^{2}+p\right)_{l i q}}{\partial x} & =p_{i n t} A \frac{\partial \alpha_{l i q}}{\partial x}+p_{l i q} \alpha_{l i q} \frac{\partial A}{\partial x} \\
& +A \lambda\left(u_{v a p}-u_{l i q}\right) \\
& -\Gamma A_{\text {int }} u_{i n t} A \\
& -F_{\text {wall friction }, l i q}-F_{\text {friction }, v a p} \\
& +(\alpha \rho)_{l i q} A \boldsymbol{g} \cdot \hat{\boldsymbol{n}}_{\text {axis }}
\end{aligned}
$$




$$
\begin{aligned}
\frac{\partial(\alpha \rho E)_{l i q} A}{\partial t}+\frac{\partial \alpha_{l i q} u_{l i q} A(\rho E+p)_{l i q}}{\partial x} & =p_{i n t} u_{i n t} A \frac{\partial \alpha_{l i q}}{\partial x}-\bar{p}_{i n t} A \mu\left(p_{l i q}-p_{\text {vap }}\right) \\
& +\bar{u}_{i n t} A \lambda\left(u_{\text {vap }}-u_{l i q}\right) \\
& +\Gamma A_{\text {int }}\left(\frac{p_{i n t}}{\rho_{\text {int }}}-H_{l i q, i n t}\right) A \\
& +Q_{i n t, l i q}+Q_{\text {wall }, \text { liq }} \\
\frac{\partial \alpha_{l i q} A}{\partial t}+u_{i n t} A \frac{\partial \alpha_{l i q}}{\partial x} & =A \mu\left(p_{l i q}-p_{\text {vap }}\right)-\frac{\Gamma A_{i n t} A}{\rho_{i n t}}
\end{aligned}
$$

for the liquid phase, and

$$
\begin{aligned}
\frac{\partial(\alpha \rho)_{\text {vap }} A}{\partial t}+\frac{\partial(\alpha \rho u)_{\text {vap }} A}{\partial x} & =\Gamma A_{\text {int }} A \\
\frac{\partial(\alpha \rho u)_{\text {vap }} A}{\partial t}+\frac{\partial \alpha_{\text {vap }} A\left(\rho u^{2}+p\right)_{\text {vap }}}{\partial x} & =p_{\text {int }} A \frac{\partial \alpha_{\text {vap }}}{\partial x}+p_{\text {vap }} \alpha_{\text {vap }} \frac{\partial A}{\partial x} \\
& +A \lambda\left(u_{\text {liq }}-u_{\text {vap }}\right) \\
& +\Gamma A_{\text {int }} u_{\text {int }} A \\
& -F_{\text {wall friction, vap }}-F_{\text {friction }, \text { liq }} \\
& +(\alpha \rho)_{\text {vap }} A \boldsymbol{g} \cdot \hat{\boldsymbol{n}}_{\text {axis }} \\
\frac{\partial(\alpha \rho E)_{\text {vap }} A}{\partial t}+\frac{\partial \alpha_{\text {vap }} u_{\text {vap }} A(\rho E+p)_{\text {vap }}}{\partial x}= & p_{\text {int }} u_{\text {int }} A \frac{\partial \alpha_{\text {vap }}}{\partial x}-\bar{p}_{\text {int }} A \mu\left(p_{\text {vap }}-p_{\text {liq }}\right) \\
+ & \bar{u}_{\text {int }} A \lambda\left(u_{\text {liq }}-u_{\text {vap }}\right) \\
- & \Gamma A_{\text {int }}\left(\frac{p_{\text {int }}}{\rho_{\text {int }}}-H_{\text {vap }, \text { int }}\right) A \\
+ & Q_{\text {int }, \text { vap }}+Q_{\text {wall }, \text { vap }} \\
\frac{\partial \alpha_{\text {vap }} A}{\partial t}+u_{\text {int }} A \frac{\partial \alpha_{\text {vap }}}{\partial x}= & A \mu\left(p_{\text {vap }}-p_{\text {liq }}\right)+\frac{\Gamma A_{\text {int }} A}{\rho_{\text {int }}}
\end{aligned}
$$

for the vapor phase. It is noted that for two-phase flow, either of the differential relations (20) or (24) may be replaced with the algebraic relation

$$
\alpha_{v a p}=1-\alpha_{l i q}
$$

throughout, reducing the total number of equations to be solved to seven.

In equations (17)-(24), $\Gamma$ is the net mass transfer per unit interfacial area from the liquid to the vapor phase and $A_{\text {int }}$ is the interfacial area per unit volume of mixture. Also, 
$H_{\text {liq,int }}$ and $H_{\text {vap,int }}$ are the liquid and gas total enthalpies at the interface, respectively. The nomenclature has also been modified so that now $u_{\text {int }}$ and $\bar{u}_{\text {int }}$ are, respectively, the interfacial velocity and average interfacial velocity; and $p_{i n t}$ and $\bar{p}_{i n t}$ are, respectively, the interfacial pressure and average interfacial pressure. In the momentum balance equations $\hat{\boldsymbol{n}}_{\text {axis }}$ is the unit vector directly along the axis of the duct, which is also the \pm flow direction. Of course $F_{\text {wall friction, } k}$ is the frictional force due to the wall acting on phase $k$ and $F_{\text {friction, } k^{\prime}}$ is the frictional force acting on phase $k$ due to the presence of the other phase $k^{\prime}$. Similarly, $Q_{i n t, k}$ is the direct heat transfer from the interface to phase $k$ and $Q_{\text {wall }, k}$ is the direct heat transfer from the wall to phase $k$.

Equation system (17)-(24) is the basic system solved with RELAP-7. The system was implemented within the MOOSE computational framework following a series of logically-complete steps [5] designed to confidently allow physically- and mathematicallymeaningful benchmark testing at each step of increased complexity. This 7-equation twophase model allows both phases to be compressible.

\subsection{Seven-Equation Two-Phase Flow Constitutive Models}

Without additional closure equations the balance relations derived above are generic, i.e. they apply to all materials (fluids). They must made to apply to the unique material (fluid) being considered - material specific. Also, though averaging the microlevel balance equations led to the "simplified" or perhaps more tractable model above, this simplification (averaging) led to a loss of information, and some additional relations must also be specified to supply (or restore) at least some information that was lost in this process ${ }^{1}$. Collectively, any additional relations, or sub-models, that must be specified to render mathematical closure (allowing a solution to be obtainable) to the generic balance equations are known as "constitutive relations".

Because the 7-equation two-phase model's most unique features are reflected in the presence of a volume fraction evolution equation, interfacial pressure and velocity, and mechanical relaxation terms involving pressure and velocity relaxation, it is natural to begin with their constitutive relations. Constitutive ideas associated with the volume fraction evolution equation were discussed previously for pedagogical reasons. Thermodynamical relaxation will be discussed subsequently, followed by other closures.

\footnotetext{
${ }^{1}$ The process of averaging the balance equations produced a system with more unknowns than equations; thus postulates or empirical correlations are required to resolve this deficiency.
} 


\subsubsection{Interface Pressure and Velocity, Mechanical Relaxation Coefficients}

In the continuous limit of small mesh spacing and time steps along with employment of the Godunov weak wave limit, the finite closure relations converge $[6,7]$ to

$$
\begin{aligned}
& p_{i n t}=\bar{p}_{i n t}+\frac{Z_{l i q} Z_{v a p}}{Z_{l i q}+Z_{v a p}} \operatorname{sgn}\left(\frac{\partial \alpha_{l i q}}{\partial x}\right)\left(u_{v a p}-u_{l i q}\right) \\
& \bar{p}_{\text {int }}=\frac{Z_{v a p} p_{l i q}+Z_{l i q} p_{v a p}}{Z_{l i q}+Z_{v a p}} \\
& u_{i n t}=\bar{u}_{i n t}+\operatorname{sgn}\left(\frac{\partial \alpha_{l i q}}{\partial x}\right) \frac{p_{v a p}-p_{l i q}}{Z_{l i q}+Z_{v a p}} \\
& \bar{u}_{i n t}=\frac{Z_{l i q} u_{l i q}+Z_{v a p} u_{v a p}}{Z_{l i q}+Z_{v a p}} \\
& \lambda=\frac{1}{2} \mu Z_{l i q} Z_{v a p} \\
& \mu=\frac{A_{\text {int }}}{Z_{\text {liq }}+Z_{\text {vap }}}
\end{aligned}
$$

where $\lambda$ is the velocity relaxation coefficient function, $\mu$ is the pressure relaxation coefficient function, $Z_{k}=\rho_{k} c_{k},(k=l i q, v a p)$, is the phasic acoustic impedance and $A_{\text {int }}$ is the specific interfacial area (i.e. the interfacial surface area per unit volume of two-phase mixture) which must be specified from some type of flow regime map or function. The DEM model for two-phase flow of water and its vapor in a one dimensional duct of spatially varying cross-section was derived and demonstrated with these closures by Berry et al. [8].

Remark (1): From this specification of $\lambda$ and $\mu$ it is clear that special coupling is rendered. To relax the 7-equation model to the ill-posed classical 6-equation model, the pressures should be relaxed toward a single pressure for both phases. This is accomplished by specifying the pressure relaxation coefficient to be very large, i.e. letting it approach infinity. But if the pressure relaxation coefficient goes to infinity, so does the velocity relaxation rate also approach infinity. This then relaxes the 7-equation model not to the classical 6-equation model, but to the mechanical equilibrium 5-equation model of Kapila. This reduced 5-equation model is also hyperbolic and well-posed. The 5-equation model provides a very useful starting point for constructing multi-dimensional interface resolving methods which dynamically captures evolving, and even spontaneously generating, 
interfaces [9]. Thus the 7-equation model of RELAP-7 can be relaxed locally to couple seamlessly with such a multi-dimensional, interface resolving code.

Remark (2): Numerically, the mechanical relaxation coefficients $\mu$ (pressure) and $\lambda$ (velocity) can be relaxed independently to yield solutions to useful, reduced models (as explained previously). It is noted, however, that relaxation of pressure only by making $\mu$ large without relaxing velocity will indeed give ill-posed and unstable numerical solutions, just as the classical 6-equation two-phase model does, with sufficiently fine spatial resolution, as confirmed in $[8,10]$.

Remark (3): Even though the implementation of the 7-equation two-phase model within RELAP-7 (or any other code for that matter) does not use the generalized approach of DEM, the interfacial pressure and velocity closures as well as the pressure and velocity relaxation coefficients of Equations (26) to (31) are utilized.

\subsubsection{Wall and Interphase Friction}

A simple wall friction model results from making the same assumptions as for singlephase duct flow with the exception that the duct wall area over which the shear stress acts is reduced by the fraction of the wall area which the phase occupies. Thus

$$
F_{\text {wall friction }, k}=\frac{f_{k}}{2 d_{h}} \rho_{k} u_{k}\left|u_{k}\right| \alpha_{k} A
$$

for phases $k=(l i q, v a p)$, where $f_{k}$ is the wall friction factor associated with phase $k$. The hydraulic diameter $d_{h}$ depends on the shape of the cross section, and the position $x$ in the pipe.

The friction force acting between the two phases due to their relative motion is also given in analogy to that of single-phase duct flow:

$$
F_{\text {friction, } k^{\prime}}=f_{k, k^{\prime}} \frac{1}{2} \rho_{k}\left(u_{k}-u_{\text {int }}\right)\left|u_{k}-u_{\text {int }}\right| A_{\text {int }} A
$$

for $k=(l i q, v a p), k^{\prime}=(\operatorname{vap}, \operatorname{liq})$, with $f_{k, k^{\prime}}$ denoting the friction factor acting upon phase $k$ due to the (relative) motion of the other phase $k^{\prime}$.

The frictional pressure drop in each phase will be different in general due the different velocities of the two phases. However, because of the tendency toward pressure equilibrium between the phases an effective pressure drop will be realized. 


\subsubsection{Wall and Interface Direct Heat Transfer}

Without wall boiling, a simple model for the direct, convective heat transfer from the wall to fluid phase $k$ will be the same as that of a single-phase except the duct wall area over which this heat transfer can occur is weighted by the wetted fraction of the phase. That is,

$$
Q_{\mathrm{wall}, k}=H_{w, k} a_{w}\left(T_{k}-T_{\mathrm{wall}}\right) \alpha_{k} A
$$

for phase $k=(l i q, v a p)$, where $H_{w, k}$ is the wall convective wall heat transfer coefficient associated with phase $k$. Similarly, the direct heat transfer from/to the interface to/from the phase $k$, which will also be used to determine the mass transfer between the phases, is

$$
Q_{\text {int }, k}=h_{T, k}\left(T_{i n t}-T_{k}\right) A_{i n t} A
$$

with $h_{T, k}$ denoting the convective heat transfer coefficient between the interface and phase $k$. The phasic bulk temperature $T_{k}$ is determined from the respective phase's equation of state.

\subsubsection{Interphase Mass Transfer}

For a vapor to be formed from the liquid phase (vaporization) energy must be added to the liquid to produce vapor at nucleation sites; whether the liquid is heated directly or decompressed below its saturation pressure. A liquid to vapor phase change may occur based on two main mechanisms. The first is related to vaporization induced by external heating or heat transfer in a nearly constant pressure environment which is called heterogeneous boiling, or simply boiling. This heat input can occur through a solid/liquid interface with the solid typically hotter than the liquid, or through a liquid/gas interface with the gas being hotter than the liquid.

To examine the mass flow rate between phases, local mechanisms of the vaporization (condensation) process are considered between the liquid phase and its associated vapor in the presence of temperature gradients. The mechanisms of interest here are dominated by heat diffusion at the interface. The pertinent local equations to consider are the mass and energy equations. As a vaporization front propagates slowly (on the order of $1 \mathrm{~mm} / \mathrm{s}$ to $1 \mathrm{~m} / \mathrm{s}$ ) compared to acoustic waves present in the medium (which propagate with speeds of the order $1 \mathrm{~km} / \mathrm{s}$ ), acoustic propagation results in quasi-isobaric pressure evolution through vaporization fronts. The momentum equation is therefore not needed - 

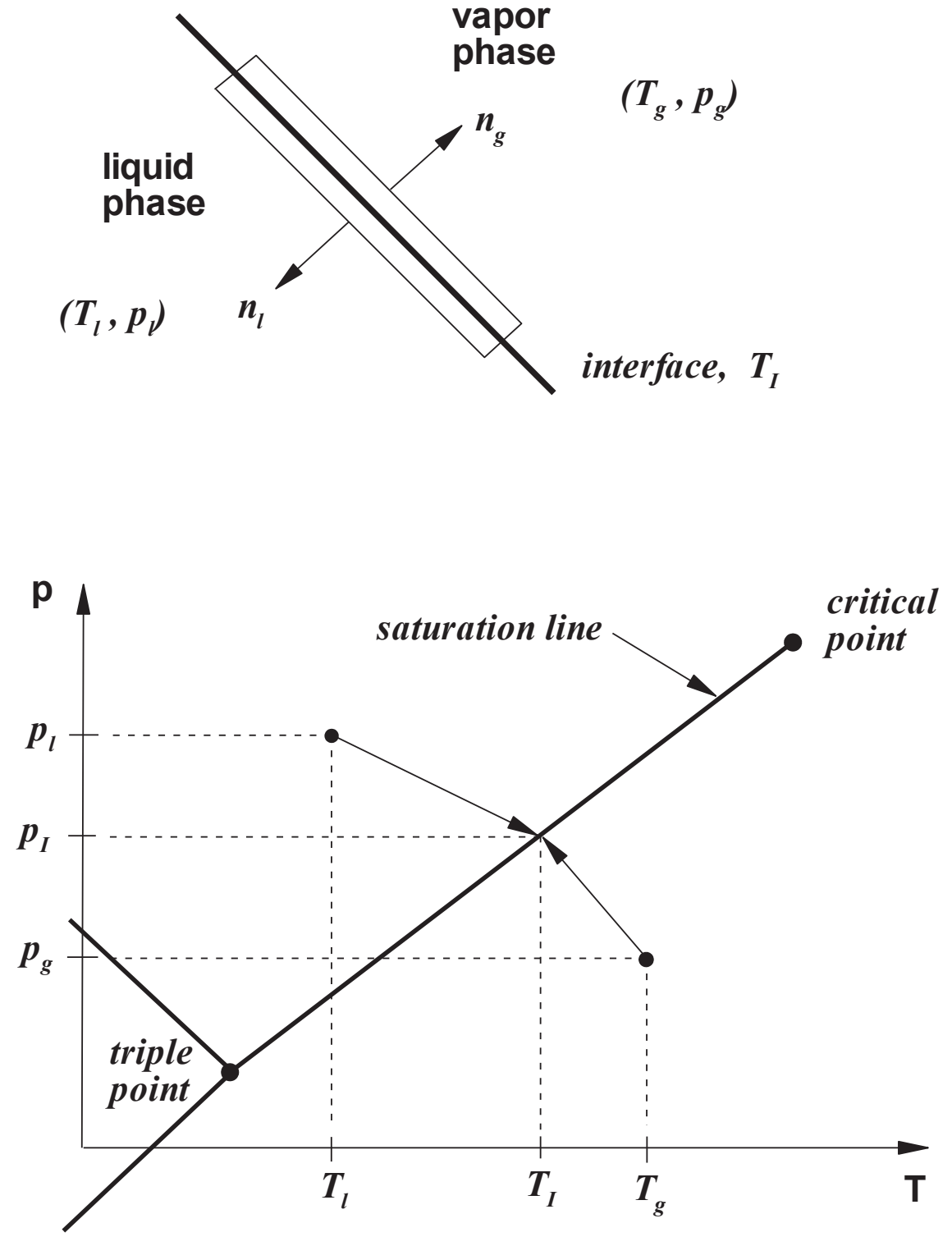

Figure 1. Interface control volume (top); $T-p$ state space around saturation line, $T_{l i q}<T_{v a p}$, (bottom). 
because the quasi-isobaric assumption (neglecting the pressure and kinetic energy variations in the total energy equation) is made. A simple expression for the interphase mass flow rate is obtained

$$
\begin{aligned}
\Gamma=\Gamma_{\text {vap }} & =\frac{h_{T, l i q}\left(T_{l i q}-T_{i n t}\right)+h_{T, v a p}\left(T_{v a p}-T_{i n t}\right)}{h_{\text {vap }, \text { int }}-h_{\text {liq }, \text { int }}} \\
& =\frac{h_{T, l i q}\left(T_{l i q}-T_{i n t}\right)+h_{T, v a p}\left(T_{v a p}-T_{i n t}\right)}{L_{v}\left(T_{\text {int }}\right)}
\end{aligned}
$$

where $L_{v}\left(T_{\text {int }}\right)=h_{\text {vap,int }}-h_{\text {liq,int }}$ represents the latent heat of vaporization. The interface temperature is determined by the saturation constraint $T_{\text {int }}=T_{\text {sat }}(p)$ with the appropriate pressure $p=\bar{p}_{\text {int }}$ determined above, the interphase mass flow rate is thus determined. The lower graphic of Figure 1, schematically shows the $p-T$ state space in the vicinity of the saturation line (shown for the case with $T_{l i q}<T_{\text {vap }}$ ).

To better illustrate the model for vaporization or condensation, Figure 2 shows pure liquid and pure vapor regions separated by an interface. Representative temperature profiles are shown for heat transfer from vapor to liquid or liquid to vapor. As discussed by Moody [1], either vaporization or condensation can occur for both temperature profiles. The interphase mass transfer is determined by the net interfacial heat transfer: if net heat transfer is toward the interface, vapor will form; conversely, if net heat transfer is away from the interface, liquid will condense. Figure 2 shows heat transfer rates $q_{v a p}$ and $q_{l i q}$ from the vapor and liquid sides of the interface. For bidirectional phase change (vaporization and condensation), mass transfer based on heat balance at the interface is adopted. When vaporization occurs, vapor is assumed to form at a saturated interface temperature $T_{\text {int }}=T_{\text {sat }}\left(\bar{p}_{\text {int }}\right)$. If condensation occurs, liquid is assumed to form also at a saturated interface temperature $T_{\text {int }}=T_{\text {sat }}\left(\bar{p}_{\text {int }}\right)$. The interfacial total enthalpies correspond to the saturated values in order that the interphase mass transfer rate and conservation of total energy be compatible:

$$
H_{k, i n t}=h_{k, i n t}+\frac{1}{2} u_{i n t}^{2}
$$

for phase $k=($ liq, vap $)$, where $h_{k, \text { int }}$ is the phase $k$ specific enthalpy evaluated at the interface condition. Phasic specific enthalpy depends upon the equation of state used and will be discussed with the equations of state. The interfacial density corresponds to the liquid saturated density $\rho_{\text {int }}=\rho_{\text {liq,sat }}\left(p_{\text {int }}\right)$. 

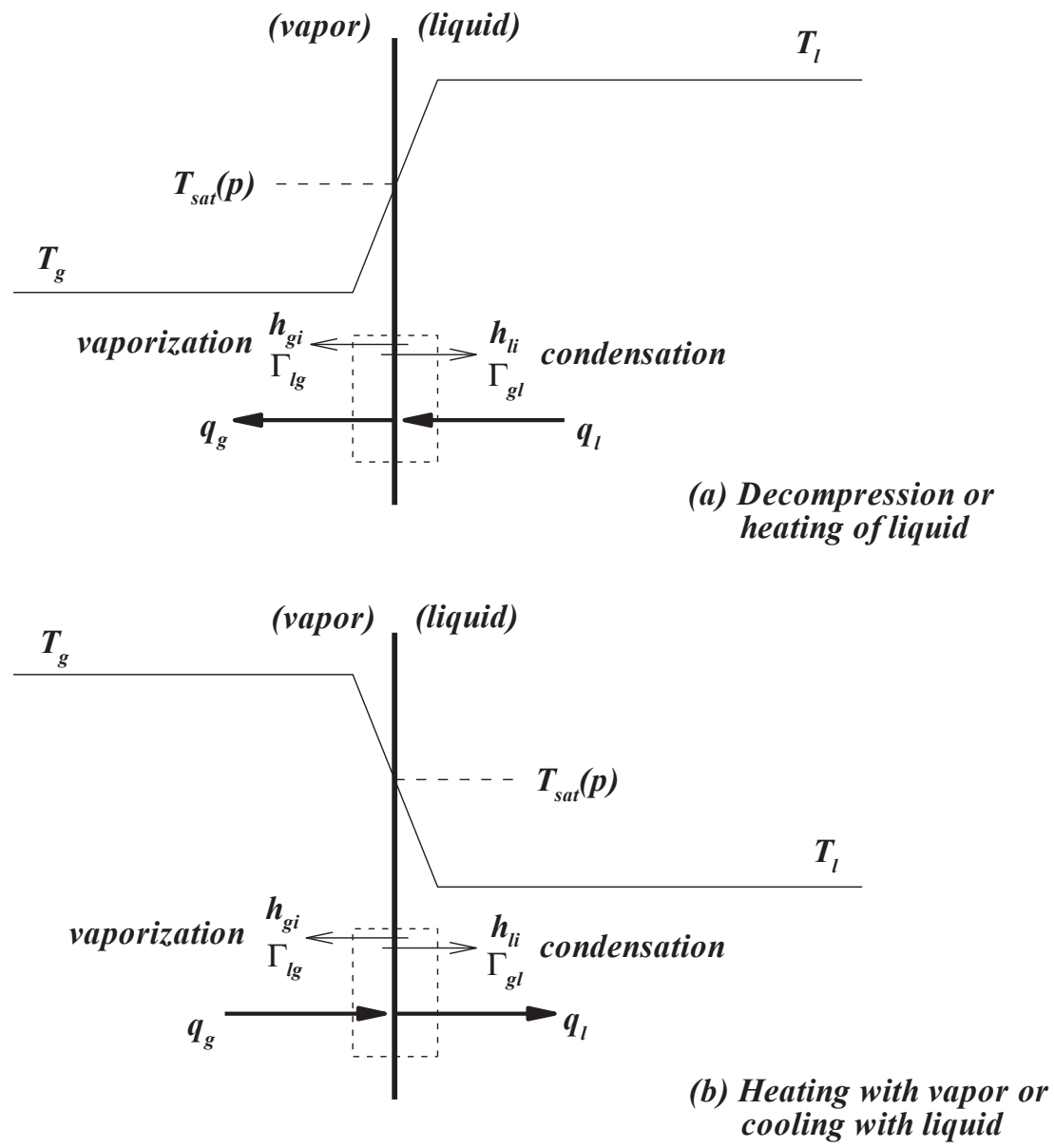

Figure 2. Vaporization and condensation at a liquid-vapor interface (after Moody [1]). 


\subsubsection{Stiffened Gas Equation of State for Two-phase Flows}

With the 7-equation two-phase model each phase is compressible and behaves with its own convex equation of state (EOS). For initial development purposes it was decided to use a simple form capable of capturing the essential physics. For this purpose the stiffened gas equation of state (SGEOS) [2] was selected (as it was also for single phase)

$$
p(\rho, e)=(\gamma-1) \rho(e-q)-\gamma p_{\infty}
$$

where $p, \rho, e$, and $q$ are the pressure, density, internal energy, and the binding energy of the fluid considered. The parameters $\gamma, q$, and $p_{\infty}$ are the constants (coefficients) of each fluid. The first term on the right hand side is a repulsive effect that is present for any state (gas, liquid, or solid), and is due to molecular vibrations. The second term on the right represents the attractive molecular effect that guarantees the cohesion of matter in the liquid or solid phases. The parameters used in this SGEOS are determined by using a reference curve, usually in the $\left(p, \frac{1}{\rho}\right)$ plane.

To extend this equation of state for two phases, LeMetayer [2] uses the saturation curves as this reference curve to determine the stiffened gas parameters for liquid and vapor phases. The SGEOS is the simplest prototype that contains the main physical properties of pure fluids, repulsive and attractive molecular effects, thereby facilitating the handling of the essential physics and thermodynamics with a simple analytical formulation. Thus each fluid has its own thermodynamics. For each phase the thermodynamic state is determined by the SGEOS:

$$
\begin{aligned}
e(p, \rho) & =\frac{p+\gamma p_{\infty}}{(\gamma-1) \rho}+q \\
\rho(p, T) & =\frac{p+p_{\infty}}{(\gamma-1) c_{v} T} \\
h(T) & =\gamma c_{v} T+q \\
g(p, T) & =\left(\gamma c_{v}-q^{\prime}\right) T-c_{v} T \ln \frac{T^{\gamma}}{\left(p+p_{\infty}\right)^{\gamma-1}}+q
\end{aligned}
$$

where $T, h$, and $g$ are the temperature, enthalpy, and Gibbs free enthalpy, respectively, of the phase considered. In addition to the three material constants mentioned above, two additional material constants have been introduced, the constant volume specific heat $c_{v}$ and the parameter $q^{\prime}$. The method to determine these parameters in liquid-vapor systems, and in particular the coupling of liquid and vapor parameters, is given in [2]. The values for water and its vapor from that reference are given in Table 2. These parameter values 
appear to yield reasonable approximations over a temperature range from 298 to $473 \mathrm{~K}$. For higher temperature range the parameters can easily be refit.

Unlike van der Waals type modeling where mass transfer is a thermodynamic path, with the 7-equation two-phase model the mass transfer modeling, which produces a relaxation toward thermodynamic equilibrium, is achieved by a kinetic process. Thus the 7-equation model preserves hyperbolicity during mass transfer. From equation (41) it is readily seen that the phase $k$ specific enthalpy evaluated at the interface condition from equation (37) is

$$
h_{k, \text { int }}=c_{p, k} T_{\text {int }}+q_{k}
$$

because $c_{p, k}=\gamma_{k} c_{v, k}$.

The bulk interphase mass transfer from the liquid phase to the vapor phase $\Gamma$ is due to their difference in Gibb's free energy. At saturated conditions the Gibb's energies of the two-phases are equal. It is necessary to determine the saturation temperature $T_{\text {sat }}(p)$ for given pressure $p=\bar{p}_{\text {int }}$ and the heat of vaporization $L_{v}\left(T_{\text {sat }}\left(\bar{p}_{\text {int }}\right)\right)$ at this saturation temperature with the SGEOS for each phase. For this calculation the procedure of [2] is adopted. This procedure for the determination of SGEOS parameters can be made very accurate provided the two reference states are picked sufficiently close to represent the experimental saturation curves as locally quasi-linear. Restrictions occur near the critical point, but away from this point wide ranges of temperatures and pressures can be considered. At thermodynamic equilibrium at the interface, the two phasic Gibbs free enthalpies must be equal, $g_{v a p}=g_{l i q}$, so the use of equation (42) yields

$$
\ln \left(p+p_{\infty, v a p}\right)=A+\frac{B}{T}+C \ln (T)+D \ln \left(p+p_{\infty, l i q}\right)
$$

where

$$
\begin{aligned}
A & =\frac{c_{p, l i q}-c_{p, v a p}+q_{v a p}^{\prime}-q_{l i q}^{\prime}}{c_{p, v a p}-c_{v, v a p}} \\
B & =\frac{q_{l i q}-q_{v a p}}{c_{p, v a p}-c_{v, v a p}} \\
C & =\frac{c_{p, v a p}-c_{p, l i q}}{c_{p, v a p}-c_{v, v a p}} \\
D & =\frac{c_{p, l i q}-c_{v, l i q}}{c_{p, v a p}-c_{v, v a p}} .
\end{aligned}
$$

Relation (44) is nonlinear, but can used to compute the theoretical curve $T_{\text {sat }}(p)$. A simple Newton iterative numerical procedure is used. With $T_{\text {sat }}(p)$ determined, the heat of 
vaporization is calculated as

$$
\begin{aligned}
L_{v}\left(T_{i n t}\right) & =h_{\text {vap }, \text { int }}-h_{\text {liq,int }} \\
& =h_{k, \text { int }} \\
& =\left(\gamma_{\text {vap }} c_{v, v a p} T+q_{v a p}\right)-\left(\gamma_{l i q} c_{v, l i q} T+q_{l i q}\right) .
\end{aligned}
$$




\section{Numerical Methods and Entropy Viscosity Stabilization}

Because the stabilization of the numerical solution of hyperbolic equation systems is intimately connected with the temporal and spatial discretization, before discussing the entropy viscosity stabilization method used in RELAP-7, a short summary of the numerical methods it employs is given.

\subsection{Spatial Discretization Algorithm}

RELAP-7 currently employs the continuous finite element method implemented via the INL MOOSE framework. For simplicity of exposition this section focuses on the weak statement associated to the strong form of the one-dimensional, variable cross-sectional area form of the Euler equations (1)-(3) summarized in Section 2.1.1. Start by writing the equations in "vector" form as

$$
\boldsymbol{R}(\boldsymbol{U}) \equiv \frac{\partial \boldsymbol{U}}{\partial t}+\frac{\partial \boldsymbol{F}}{\partial x}-\boldsymbol{S}=\mathbf{0}
$$

where

$$
\boldsymbol{U} \equiv\left[\begin{array}{c}
\rho A \\
\rho u A \\
\rho E A
\end{array}\right], \quad \boldsymbol{F} \equiv\left[\begin{array}{c}
\rho u A \\
\left(\rho u^{2}+p\right) A \\
\rho u H A
\end{array}\right]
$$

and $\boldsymbol{S}=\boldsymbol{S}(\boldsymbol{U})$ consists of the remaining source terms. Note that $\boldsymbol{U}$ and $\boldsymbol{F}$ are identical to their meanings in the "constant-area" equations, up to multiplication by the area, $A$. The variational statement proceeds by dotting (50) by an "admissible" vector test function $W$ (more details of which will be subsequently given), integrating over the domain $\Omega$, and applying the divergence theorem. Solutions $\boldsymbol{U}$ are sought such that

$$
\int_{\Omega}\left(\frac{\partial \boldsymbol{U}}{\partial t} \cdot \boldsymbol{W}-\boldsymbol{F} \cdot \frac{\partial \boldsymbol{W}}{\partial x}-\boldsymbol{S} \cdot \boldsymbol{W}\right) \mathrm{d} \Omega+\int_{\Gamma}(\boldsymbol{F} \cdot \boldsymbol{W}) \hat{n}_{x} \mathrm{~d} \Gamma=0
$$

holds for all admissible $\boldsymbol{W}$. Note that the test function $\boldsymbol{W}$ is not chosen arbitrarily. In particular, it is required that $\boldsymbol{W}$ come from the space of vector functions

$$
\boldsymbol{W} \in\left\{\left[\begin{array}{l}
w \\
0 \\
0
\end{array}\right],\left[\begin{array}{l}
0 \\
w \\
0
\end{array}\right],\left[\begin{array}{l}
0 \\
0 \\
w
\end{array}\right]\right\}
$$


where $w \in \mathcal{W}$ is a scalar test function. In the present work, and in general practice, the space $\mathcal{W}$ is taken to be (a subspace of) the Hilbert space $H^{1}(\Omega)$. This choice, for instance, guarantees enough smoothness that (52) makes sense. The approximate problem then proceeds by selecting only test functions from a finite-dimensional subspace of $\mathcal{W}$, denoted by $\mathcal{W}^{h}$, which is spanned by the basis $\left\{\phi_{i}\right\}, i=1, \ldots, N$. We then seek $\boldsymbol{U}^{h}$ with components in the same space as $\mathcal{W}^{h}$, satisfying the boundary conditions, and such that

$$
\int_{\Omega}\left(\frac{\partial \boldsymbol{U}^{h}}{\partial t} \cdot \boldsymbol{W}^{h}-\boldsymbol{F}^{h} \cdot \frac{\partial \boldsymbol{W}^{h}}{\partial x}-\boldsymbol{S}^{h} \cdot \boldsymbol{W}^{h}\right) \mathrm{d} \Omega+\int_{\Gamma}\left(\boldsymbol{F}^{h} \cdot \boldsymbol{W}^{h}\right) \hat{n}_{x} \mathrm{~d} \Gamma=0
$$

holds for all $\boldsymbol{W}^{h}$ defined analogously to (53), with components in $\mathcal{W}^{h}$. Note that (54) has been placed in a "continuous" setting, that is, a mesh and finite element discretization has been introduced requiring a continuous solution. Equation (54) remains a "weak" restatement of the "strong" equations (50) in the sense that derivatives of the solution and its flux need not be continuous. More will be said of this subsequently, in the upcoming section on stabilization methods. Written out in component form, and denoting the components of $U^{h}$ by $U_{0}^{h}, U_{1}^{h}$, and $U_{2}^{h},(54)$ expands to:

$$
\begin{aligned}
& \int_{\Omega}\left(\frac{\partial U_{0}^{h}}{\partial t} \phi_{i}-U_{1}^{h} \frac{\partial \phi_{i}}{\partial x}\right) \mathrm{d} \Omega+\int_{\Gamma} U_{1}^{h} \hat{n}_{x} \phi_{i} \mathrm{~d} \Gamma=0 \\
& \int_{\Omega}\left[\left(\frac{\partial U_{1}^{h}}{\partial t}-U_{0}^{h} g_{x}+\frac{f}{2 d_{h}} U_{1}^{h}\left|\frac{U_{1}^{h}}{U_{0}^{h}}\right|-p^{h} \frac{\partial A}{\partial x}\right) \phi_{i}-\left(\frac{\left(U_{1}^{h}\right)^{2}}{U_{0}^{h}}+p^{h} A\right) \frac{\partial \phi_{i}}{\partial x}\right] \mathrm{d} \Omega \\
& \quad+\int_{\Gamma}\left(\frac{\left(U_{1}^{h}\right)^{2}}{U_{0}^{h}}+p^{h} A\right) \hat{n}_{x} \phi_{i} \mathrm{~d} \Gamma=0 \\
& \int_{\Omega}\left[\left(\frac{\partial U_{2}^{h}}{\partial t}+H_{w} a_{w}\left(T^{h}-T_{w}\right) A-U_{1}^{h} g_{x}\right) \phi_{i}-U_{1}^{h} H^{h} \frac{\partial \phi_{i}}{\partial x}\right] \mathrm{d} \Omega \\
& \quad+\int_{\Gamma} U_{1}^{h} H^{h} \hat{n}_{x} \phi_{i} \mathrm{~d} \Gamma=0
\end{aligned}
$$

Equations (55)-(57) must hold for $i=1, \ldots, N$. Note that the approximate pressure, $p^{h}$, temperature, $T^{h}$, and enthalpy, $H^{h}$ are functions of the conserved variables $U_{0}^{h}, U_{1}^{h}, U_{2}^{h}$. As mentioned, a continuous Galerkin formulation is employed, and therefore the unknowns 
are expressed in the same basis used for the test functions, i.e.

$$
\begin{aligned}
U_{0}^{h} & =\sum_{j}\left(U_{0}\right)_{j} \phi_{j} \\
U_{1}^{h} & =\sum_{j}\left(U_{1}\right)_{j} \phi_{j} \\
U_{2}^{h} & =\sum_{j}\left(U_{2}\right)_{j} \phi_{j}
\end{aligned}
$$

The coefficients $\left(U_{0}\right)_{j},\left(U_{1}\right)_{j}$, and $\left(U_{2}\right)_{j}$ vary in time only, and comprise the solution vector at each iteration. Note that (55)-(57) are so-called "semi-discrete" equations: they have been discretized in space, but the temporal derivatives remain in continuous form. In Section 4.2 the various time discretization methods employed in RELAP-7, to approximately time-integrate the equations, are summarized. Furthermore, it is well-known that a continuous Galerkin discretization of this set of hyperbolic equations is equivalent to a central difference method for a certain choice of integration rule, and therefore will exhibit oscillatory instabilities unless some artificial diffusion is added to stabilize the method. In Section 4.4, stabilization will be discussed further, and in particular the new entropy viscosity stabilization scheme will be presented in detail.

\subsection{Time Integration Methods}

RELAP-7, through MOOSE, supports a number of standard implicit time integration methods such as the backward Euler (Section 4.2.1) and BDF2 (Section 4.2.2) methods.

\subsubsection{Backward Euler}

The backward Euler method [11] is a well-known, first-order, A-stable implicit time integration method. Given a generic semi-discrete equation in a form similar to (55)-(57),

$$
\int_{\Omega}\left(\frac{\partial u^{h}}{\partial t}+G\left(u^{h}\right)\right) \phi_{i} \mathrm{~d} \Omega=0
$$

the backward Euler method results in the temporal discretization

$$
\int_{\Omega}\left(\frac{u^{n+1}-u^{n}}{\Delta t}+G\left(u^{n+1}\right)\right) \phi_{i} \mathrm{~d} \Omega=0
$$


where $\Delta t$ is the timestep, $t^{n+1}=t^{n}+\Delta t$, and $u^{n} \equiv u^{h}\left(t^{n}\right)$ is a shorthand notation used to refer to the finite element solution at time level $n$. Equation (62) is a fully-discrete (possibly nonlinear) equation which must be satisfied for each $i$.

Note that the backward Euler method, when applied to the linear convection equation

$$
\frac{\partial u}{\partial t}+a \frac{\partial u}{\partial x}=0
$$

yields a leading-order truncation error term of the form

$$
\begin{aligned}
\left.\frac{\partial u}{\partial t}\right|_{t^{n+1}} & =\frac{u^{n+1}-u^{n}}{\Delta t}+\left.\frac{\Delta t}{2} \frac{\partial^{2} u}{\partial t^{2}}\right|_{t^{n+1}}+\mathcal{O}\left(\Delta t^{2}\right) \\
& =\frac{u^{n+1}-u^{n}}{\Delta t}+\left.\frac{a^{2} \Delta t}{2} \frac{\partial^{2} u}{\partial x^{2}}\right|_{t^{n+1}}+\mathcal{O}\left(\Delta t^{2}\right)
\end{aligned}
$$

where (64) follows from differentiating the continuous equation (63) with respect to time:

$$
\frac{\partial^{2} u}{\partial t^{2}}=-a \frac{\partial}{\partial t}\left(\frac{\partial u}{\partial x}\right)=-a \frac{\partial}{\partial x}\left(\frac{\partial u}{\partial t}\right)=-a \frac{\partial}{\partial x}\left(-a \frac{\partial u}{\partial x}\right)=a^{2} \frac{\partial^{2} u}{\partial x^{2}}
$$

Rearranging terms in (64) and adding $a \frac{\partial u}{\partial x}$ to both sides allows us to write

$$
\frac{u^{n+1}-u^{n}}{\Delta t}+a \frac{\partial u}{\partial x}=\frac{\partial u}{\partial t}+a \frac{\partial u}{\partial x}-\frac{a^{2} \Delta t}{2} \frac{\partial^{2} u}{\partial x^{2}}+\mathcal{O}\left(\Delta t^{2}\right)
$$

where all the continuous derivatives are assumed to be evaluated at time level $t^{n+1}$. Thus, the semi-discrete form of the linear convection on the left-hand side of (66) is equal to the continuous parabolic partial differential equation on the right-hand side, which includes "artificial" diffusion or viscosity of $\mathcal{O}\left(\frac{a^{2} \Delta t}{2}\right)$, to within $\mathcal{O}\left(\Delta t^{2}\right)$. For this reason, we often say that the backward Euler time discretization is inherently stabilizing for the hyperbolic equation (63). Obviously, the artificial viscosity for the complete scheme is a composite of the artificial viscosity of both the time and spatial discretization.

The backward Euler time integration method may generate excessive artificial viscosity and should, therefore, only be used for transients with RELAP-7 as an initial scoping calculation, or if only the steady-state solution is of interest. For accurate transient solutions with RELAP-7, the BDF2 time integration method, described next, is highly recommended because it is a second-order (in time) discretization. 


\subsubsection{BDF2}

The backward differentiation formula (BDF) is a family of implicit methods for numerically integrating ordinary differential equations. Some notable members of this family include BDF1, which is equivalent to the backward Euler [12] method discussed in Section 4.2.1, and BDF2, which is the highest-order BDF method which is still A-stable. For fixed step-size $\Delta t$, the BDF2 method applied to the ordinary differential equation

$$
\begin{aligned}
\frac{\partial u}{\partial t} & =f(t, u) \\
u(t=0) & =u_{0}
\end{aligned}
$$

yields the update step:

$$
u^{n+1}=\frac{4}{3} u^{n}-\frac{1}{3} u^{n-1}+\frac{2}{3} \Delta t f\left(u^{n+1}, t^{n+1}\right)
$$

Dividing through by $\frac{2}{3} \Delta t$, equation (69) can be alternatively written as

$$
\frac{\frac{3}{2} u^{n+1}-2 u^{n}+\frac{1}{2} u^{n-1}}{\Delta t}=f\left(u^{n+1}, t^{n+1}\right)
$$

The left-hand side of (70) can be interpreted as a backward-difference approximation to the continuous time derivative $\frac{\partial u}{\partial t}$, and may be employed in a manner analogous to (62) to derive a fully-discrete system of equations:

$$
\int_{\Omega}\left(\frac{\frac{3}{2} u^{n+1}-2 u^{n}+\frac{1}{2} u^{n-1}}{\Delta t}+G\left(u^{n+1}\right)\right) \phi_{i} \mathrm{~d} \Omega=0
$$

based on the semi-discrete equations (55)-(57). Since BDF2 requires two old timesteps, the method must be "bootstrapped" by a lower-order method, such as backward Euler, when starting. This means that a much smaller time step size should be used for start-up, at the beginning of a transient. The BDF2 method is recommended for most transient simulations with RELAP-7.

\subsection{Jacobian-Free Newton Krylov Solver}

The RELAP-7 code solves coupled multi-physics problems using the Jacobian-Free Newton Krylov (JFNK) approach via the MOOSE framework. Field equations solved in 
the current RELAP-7 code include PDEs to describe one-dimensional fluid flow in pipe systems and heat conduction in solids, as well as ODEs to describe physics in zerodimensional components and the point kinetics equations.

The JFNK method is a fully-coupled, multi-level method for solving large nonlinear equation systems. In general, it consists of at least two levels: the outer Newton loop for the nonlinear solve and the inner Krylov loop for the linear systems of equations associated to Newton iteration. The JFNK method has become an increasingly popular option for solving large nonlinear equation systems arising from multi-physics problems over the last 20 years, and has branched out into a number of different disciplines [13].

In what follows, a brief description of the JFNK method as it applies to the RELAP-7 application is given. The FEM-discretized field equations are first written as

$$
\boldsymbol{F}(\boldsymbol{u})=\mathbf{0}
$$

where $\boldsymbol{F}$ represents the nonlinear equation system and $\boldsymbol{u}$ is the solution vector. Newton's method requires an initial guess, $\boldsymbol{u}^{0}$, to start the iteration process. For the transient problems of interest here, the solution at a previous time step is generally used as the initial guess for the method. At the $k^{t h}$ iteration, the residual vector is defined as

$$
\boldsymbol{r}^{k} \equiv \boldsymbol{F}\left(\boldsymbol{u}^{k}\right)
$$

Clearly if $\boldsymbol{u}^{k}$ satisfies (72) exactly, the $k^{\text {th }}$ residual will be zero. To update the solution vector, the following equation is solved for the update vector, $\delta \boldsymbol{u}^{k+1}$ :

$$
\boldsymbol{J}\left(\boldsymbol{u}^{k}\right) \delta \boldsymbol{u}^{k+1}=-\boldsymbol{r}^{k}
$$

where $\boldsymbol{J}\left(\boldsymbol{u}^{k}\right)$ is the Jacobian matrix evaluated at $\boldsymbol{u}^{k}$. In index notation,

$$
J_{i j} \equiv \frac{\partial F_{i}}{\partial u_{j}} .
$$

After $\delta \boldsymbol{u}^{k+1}$ is obtained, the $(k+1)^{s t}$ solution iterate is computed by

$$
\boldsymbol{u}^{k+1}=\boldsymbol{u}^{k}+\delta \boldsymbol{u}^{k+1}
$$

The Newton iteration is terminated when one of the following conditions is met:

1. The residual vector norm, $\left|\boldsymbol{r}^{k}\right|$, is sufficiently small. 
2. The relative residual vector norm $\frac{\left|\boldsymbol{r}^{k}\right|}{\left|\boldsymbol{r}^{0}\right|}$ is sufficiently small.

3. The step size norm, $\left|\delta \boldsymbol{u}^{k+1}\right|$ is sufficiently small.

Note that (74) represents a large linear system of equations. In the JFNK method, we need not explicitly form the matrix $\boldsymbol{J}$ : only its action on a vector (via matrix-vector product) is required. Effective preconditioning is generally required for Krylov subspace methods to be efficient, i.e., for the method to converge in a reasonable number of iterations. A preconditioned version of equation (74) can be expressed as (using right preconditioning as an example),

$$
\boldsymbol{J}^{k} \boldsymbol{P}^{-1}\left(\boldsymbol{P} \delta \boldsymbol{u}^{k+1}\right)=-\boldsymbol{r}^{k}
$$

where $\boldsymbol{P}$ is the preconditioning matrix. In the approach current used in RELAP-7, an analytical Jacobian matrix is computed according to (75), and passed to the underlying numerical solver library as the matrix $\boldsymbol{P}$ for preconditioning purposes.

\subsection{Entropy Viscosity Method}

In review of solutions to nonlinear hyperbolic, initial-boundary value problems such as the single- and two-phase equation systems of RELAP-7, it is known that even with smooth initial data, the existence of a globally smooth solution may be violated because of the nonlinearity of the flux functions and other nonlinear terms. The concept of a weak solution is introduced to guarantee the existence of a global solution; however, the uniqueness of the solution(s) is lost because the problem may allow infinitely many weak solutions. An additional condition is usually imposed, which is called the "entropy condition," to select a unique solution from the infinitely many weak solutions. More specifically, it allows solutions that are mathematically permissible, but which are not satisfactory from a physical viewpoint, to be eliminated. The unique solution is called the "entropy solution."

The term "entropy principle" was introduced by Courant and Friedrichs [14]. To justify the entropy principle, consider that a discontinuous solution may be viewed as the limit case of a continuous profile for which both sides of the discontinuity are connected to each other within a very short distance $\delta$. Satisfaction of the entropy condition insures that this continuous approximation of the nonlinear discontinuity occurs only if the tail of the wave travels faster than the front. Furthermore, it insures the speed of the discontinuity is between the wave speed of the tail and that of the front of the discontinuity. Consequently, backward discontinuities, i.e. "rarefaction shocks" are eliminated as being unphysical even 
though they are mathematically admissible (a legitimate solution). Thus, when the wave speed on the "high" side of the discontinuity is larger (in the direction from solution high to solution low) than the wave speed on the "low" side of the discontinuity, the profile becomes steeper and a discontinuity appears. On the other hand, when the wave speed on the "high" side of the discontinuity is smaller (in the direction from solution high to solution low) than the wave speed on the "low" side, the profile becomes smoother.

In the literature, although there are several different ways of defining the entropy condition, they are all equivalent in the sense that they select the same entropy solution. For numerical schemes, this entropy condition and solution is sought through utilization of so-called conservative formulations of the physically descriptive equations along with appropriate specification of an artificial viscosity, either added directly to the governing equations or implied by the discretization employed, to render a continuous profile. This artificial viscosity is tailored such that the entropy production of the "viscous" continuous profile matches sufficiently the entropy production of the discontinuity it is designed to approximate in a manner such that a monotonic representation of the discontinuity, with minimal thickness, is achieved. Essentially, a discretization scheme is selected, or built, which is consistent with the entropy condition, thereby guaranteeing that the numerical computation faithfully captures the physically relevant solution.

It is not easy to satisfy the somewhat contradictory objectives of capturing singularities (like shocks or interfaces) without instability or numerical dispersion while also realizing better resolution where the solution is smooth. Consequently, a plethora of schemes fill the literature, all attempting to accomplish this, either better or more robustly. First order Godunov upwind schemes are overly dissipative while sophisticated higher order methods, which are typically a nonlinear combination of first order dissipative schemes and basic higher order schemes that are necessarily oscillatory, need to employ flux limiters to prevent unphysical oscillations. Even linear hyperbolic equation systems can be problematic for numerical discretization schemes. For example, the well-known central difference method generally produces oscillations for simple linear advection.

It is well-known that the continuous Galerkin finite element method, used in RELAP7 is unstable, when applied directly to hyperbolic systems of equations. It attempts to approximate potentially nonlinear discontinuous solutions with continuous, $\delta$-mollified solutions as nearly as possible with the functional space selected and element spacing chosen [15]. For certain finite element spaces and integration rules, the central difference method and Galerkin finite element methods are equivalent. This spatial discretization is known to not produce sufficient entropy locally. To compensate, especially for equations in conservative form, the method attempts to achieve this through a train of entropy pro- 
ducing oscillations in the vicinity of the local entropy production deficit. For example, this discretization exhibits oscillations when applied to convection-dominated flows.

Currently available options of solution stabilization for RELAP-7 application include Streamline Upwind/Petrov Galerkin method (SUPG), Lapidus, and entropy viscosity methodologies. Plus, the low-order backward Euler time integration methods described above is known to introduce $\mathcal{O}(\Delta t)$ artificial viscosity through its discretization error. The details of the SUPG and Lapidus artificial viscosity methods are well discussed in the literature. The entropy viscosity method is new so details of the entropy viscosity method are described next.

As an available option, RELAP-7 employs a recently introduced technique [16-19], known as the entropy viscosity method, which requires the addition of artificial dissipation terms to the equations while ensuring that the physical entropy minimum principle remains satisfied. Additional details regarding its application to the 7-equation two-phase model and to low Mach number flows are directly based upon INL-sponsored, independent research of Delchini [20]. This entropy viscosity method is independent of the spatial discretization employed, so it can be used with the standard Galerkin, continuous Finite Element Method (FEM). Though shown below for a simplified 7-equation two-phase model, the entropy viscosity method is available for use with single-phase flow systems as well.

The simplified 7-equation two-phase model equation system is:

$$
\begin{aligned}
\frac{\partial}{\partial t}\left(\alpha_{k} A\right)+u_{I} A \frac{\partial \alpha_{k}}{\partial x} & =A \mu\left(P_{k}-P_{j}\right) \\
\frac{\partial}{\partial t}\left(\alpha_{k} \rho_{k} A\right)+\frac{\partial}{\partial x}\left(\alpha_{k} \rho_{k} u_{k} A\right) & =0 \\
\frac{\partial}{\partial t}\left(\alpha_{k} \rho_{k} u_{k} A\right)+\frac{\partial}{\partial x}\left[\alpha_{k} A\left(\rho_{k} u_{k}^{2}+P_{k}\right)\right] & =\alpha_{k} P_{k} \frac{\partial A}{\partial x}+P_{I} A \frac{\partial \alpha_{k}}{\partial x} \\
& +A \lambda\left(u_{j}-u_{k}\right) \\
\frac{\partial}{\partial t}\left(\alpha_{k} \rho_{k} E_{k} A\right)+\frac{\partial}{\partial x}\left[\alpha_{k} A u_{k}\left(\rho_{k} E_{k}+P_{k}\right)\right] & =P_{I} u_{I} A \frac{\partial \alpha_{k}}{\partial x}-\mu \bar{P}_{I}\left(P_{k}-P_{j}\right) \\
& +\bar{u}_{I} A \lambda\left(u_{j}-u_{k}\right)
\end{aligned}
$$

where $\rho_{k}, u_{k}, E_{k}$ and $P_{k}$ are the density, the velocity, the specific total energy and the pressure of $k^{t h}$ phase, respectively. The pressure and velocity relaxation parameters are denoted by $\mu$ and $\lambda$, respectively. The variables with index $(\cdot)_{I}$ correspond to the interfacial variables and a definition for those can be found above (and in [8]). The cross-sectional area $A$ is a function only of space, so $\frac{\partial A}{\partial t}=0$. In [8], the entropy equation is derived for each phase, by assuming that there exists a phasic entropy function $s_{k}$ that depends upon 
the density $\rho_{k}$ and the specific internal energy $e_{k}$ :

$$
\begin{aligned}
\rho_{k} A\left(\frac{\partial s_{k}}{\partial t}+u_{k} \frac{\partial s_{k}}{\partial x}\right) & =\frac{Z_{j}}{Z_{j}+Z_{k}} \lambda\left(u_{k}-u_{j}\right)^{2}+\frac{Z_{k}}{Z_{j}+Z_{k}} \mu\left(P_{k}-P_{j}\right)^{2} \\
& +\frac{Z_{k}}{Z_{j}+Z_{k}}\left|\frac{\partial \alpha}{\partial x}\right|\left[Z_{j}\left(u_{k}-u_{j}\right)+\operatorname{sgn}\left(\frac{\partial \alpha}{\partial x}\right)\left(P_{k}-P_{j}\right)\right]^{2}
\end{aligned}
$$

where $Z_{j}=\rho_{j} c_{j}$ and $c_{j}$ represents the acoustic impedance and the speed of sound of phase $j$, respectively. The $\operatorname{symbol} \operatorname{sgn}(\cdot)$ denotes a function that returns the sign of the quantity $(\cdot)$.

From (82), it is clear that the entropy minimum principle is satisfied since the righthand side is composed only of positive terms. In the remainder of this exposition all of the source terms (right-hand sides of (78)-(81)) are dropped in order to simplify the derivation. It will not affect the final result, provided that the definitions of the entropy residual (below) are amended to include contributions of any heat source/sink terms [21], since all of the source terms combine in a sum of positive terms when deriving the entropy function. The phase index $k$ is also dropped.

To apply the entropy viscosity method, appropriate dissipative terms are added to each of the equations as follows

$$
\begin{aligned}
\frac{\partial}{\partial t}(\alpha A)+u_{I} A \frac{\partial \alpha}{\partial x} & =\frac{\partial}{\partial x}(A l) \\
\frac{\partial}{\partial t}(\alpha \rho A)+\frac{\partial}{\partial x}(\alpha \rho u A) & =\frac{\partial}{\partial x}(A f) \\
\frac{\partial}{\partial t}(\alpha \rho u A)+\frac{\partial}{\partial x}\left[\alpha A\left(\rho u^{2}+P\right)\right] & =\alpha P \frac{\partial A}{\partial x}+\frac{\partial}{\partial x}(A g) \\
\frac{\partial}{\partial t}(\alpha \rho E A)+\frac{\partial}{\partial x}[\alpha A u(\rho E+P)] & =\frac{\partial}{\partial x}[A(h+u g)]
\end{aligned}
$$

where $f, g, h$ and $l$ are the dissipative terms. By adding these dissipative terms to each equation, the entropy equation gets modified; extra terms will appear in the right-hand side that are a function of the dissipative terms. The sign of these new terms needs to be studied in order to preserve positivity of the right-hand side. This is achieved by the following steps:

- Recast the system of equation given in (83)-(86) in terms of the primitive variables $(\alpha, \rho, u, e)$ (we only account for the dissipative terms here). 
- Derive the entropy equation by using the chain rule

$$
\frac{d s}{d t}=s_{\rho} \frac{d \rho}{d t}+s_{e} \frac{d e}{d t}
$$

where $\frac{d}{d t}$ is the material derivative. The terms $s_{e}$ and $s_{\rho}$ denote the partial derivative of the entropy $s$ with respect to $e$ and $\rho$, respectively.

- Isolate the terms of interest and choose an appropriate expression for each of the dissipative terms in order to ensure positivity of the right-hand side.

The first step consists of recasting the system of equations in terms of the primitive variables as

$$
\begin{aligned}
A \frac{\partial \alpha}{\partial t}+u_{I} A \frac{\partial \alpha}{\partial x} & =\frac{\partial}{\partial x}(A l) \\
\alpha A\left(\frac{\partial \rho}{\partial t}+u \frac{\partial \rho}{\partial x}\right)+\rho \alpha \frac{\partial}{\partial x}(u A)+\Upsilon & =\frac{\partial}{\partial x}(A f)-\rho \frac{\partial}{\partial x}(A l) \\
\alpha \rho A\left(\frac{\partial u}{\partial t}+u \frac{\partial u}{\partial x}\right)+\frac{\partial}{\partial x}(\alpha P A) & =\alpha P \frac{\partial A}{\partial x}+\frac{\partial}{\partial x}(A g) \\
& -u \frac{\partial}{\partial x}(A f) \\
\alpha \rho A\left(\frac{\partial e}{\partial t}+u \frac{\partial e}{\partial x}\right)+\alpha P A \frac{\partial u}{\partial x}+\alpha u P \frac{\partial A}{\partial x} & =\frac{\partial}{\partial x}(A h)+A g \frac{\partial u}{\partial x} \\
& +\left(\frac{u^{2}}{2}-e\right) \frac{\partial}{\partial x}(A f)
\end{aligned}
$$

where $\Upsilon=\rho A\left(u-u_{I}\right) \frac{\partial \alpha}{\partial x}$ has been used. The function $\Upsilon$ can be ignored since it is used to get the right-hand side of (82). For the second step, the continuity and internal energy equations can be combined using the chain rule given in (87) to obtain the entropy equation

$$
\begin{aligned}
\alpha \rho A \frac{d s}{d t}+\alpha\left(\rho^{2} s_{\rho}+P s_{e}\right) & \left(A \frac{\partial u}{\partial x}+u \frac{\partial A}{\partial x}\right)= \\
s_{e} & {\left[\frac{\partial}{\partial x}(A h)+A g \frac{\partial u}{\partial x}+\left(\frac{u^{2}}{2}-e\right) \frac{\partial}{\partial x}(A f)\right] } \\
+ & \rho s_{\rho}\left[\frac{\partial}{\partial x}(A f)-\rho \frac{\partial}{\partial x}(A l)\right] .
\end{aligned}
$$


The last step is a little more involved. For clarity of a first approach, the entropy equation (92) can be split into four separate terms for further consideration. The first term of the left-hand side is the Lagrangian (or material) derivative of the entropy function $s$. It does not need to be modified since its sign has to be determined by looking at the other terms. The second term on the left-hand side is usually set to zero by assuming that $\rho^{2} s_{\rho}+P s_{e}=0$. Any other alternative would require the term $\rho^{2} s_{\rho}+P s_{e}$ to be function of the velocity $u$ or its derivatives, and the cross-section $A$, in order to be able to determine its sign. In addition, any entropy function, obeying the relation $\rho^{2} s_{\rho}+P s_{e}=0$, also obeys the second thermodynamic law:

$$
\begin{array}{r}
T d s=d e-\frac{P}{\rho^{2}} d \rho \\
\Downarrow \\
s_{e}=\frac{1}{T} \geq 0, \quad s_{\rho}=-s_{e} \frac{P}{\rho^{2}}
\end{array}
$$

where $T$ is the fluid temperature.

The right-hand side of (92) is more difficult to handle. It requires further assumptions in the definition of the dissipative terms $h$ and $g$. The right-hand side can be simplified by introducing the following expressions for the dissipative terms $h$ and $g$

$$
\begin{aligned}
& f=\tilde{f}+\rho l \\
& g=\alpha \rho \mu \frac{\partial u}{\partial x}+u f \\
& h=\tilde{h}-\frac{u^{2}}{2} f+\rho e l
\end{aligned}
$$

which results in

$$
\begin{gathered}
s_{e}\left[\frac{\partial}{\partial x}(A h)+A g \frac{\partial u}{\partial x}+\left(\frac{u^{2}}{2}-e\right) \frac{\partial}{\partial x}(A f)\right]= \\
\underbrace{s_{e}\left[\frac{\partial}{\partial x}(A \tilde{h})-e \frac{\partial}{\partial x}(A \tilde{f})\right]+\rho s_{\rho} \partial_{x}(A \tilde{f})}_{\mathcal{R}_{1}}+s_{e} \alpha \rho \mu\left(\frac{\partial u}{\partial x}\right)^{2} \\
+\underbrace{s_{e} \partial_{x}(A \rho e l)-s_{e} e \partial_{x}(A \rho l)+\rho s_{\rho} \partial_{x}(A \rho l)-\rho^{2} s_{\rho} \partial_{x}(A l)}_{\mathcal{R}_{2}}
\end{gathered}
$$

where $\mu$ is a positive viscosity coefficient (not to be confused with the same symbol used previously for the pressure relaxation coefficient - because it is no longer being considered, 
no ambiguity should result), and $\tilde{h}$ and $\tilde{f}$ are new dissipative terms to be determined. In (97), it is noted that the term $s_{e} \alpha \rho \mu\left(\frac{\partial}{\partial x} u\right)^{2}$ is always positive and does not need any further modification. Thus, it remains to determine the sign of $\mathcal{R}_{1}$ and $\mathcal{R}_{2}$. The second term $\mathcal{R}_{2}$ can be recast as a function of the gradient of the entropy, as follows:

$$
\mathcal{R}_{2}=\rho A l \partial_{x} s
$$

One of the assumptions made in the entropy minimum principle is that the entropy is at a minimum which implies that its gradient is null. Because of this, it follows that the term $\mathcal{R}_{2}$ is zero and thus, the entropy minimum principle is verified independently of the definition of the dissipative term $l$ used in the volume fraction equation. It will be explained later in this section how to derive the definition for $l$. We now focus on the other term $\mathcal{R}_{1}$. The first step consists of integrating by part to yield:

$$
\begin{aligned}
\mathcal{R}_{1} & =\frac{\partial}{\partial x}\left[A\left(s_{e} \tilde{h}+\left(\rho s_{\rho}-e s_{e}\right) \tilde{f}\right)\right] \\
& -\tilde{h} \frac{\partial}{\partial x}\left(s_{e}\right)-A \tilde{f} \frac{\partial}{\partial x}\left(\rho s_{\rho}-e s_{e}\right) .
\end{aligned}
$$

In this form, $\mathcal{R}_{1}$ is identical to the term obtained in the derivation of the dissipative terms for the multi-D Euler equations [22]. The sign of $\mathcal{R}_{1}$, under the conditions of assuming a convex entropy and choosing the following definition for the dissipative terms $\tilde{h}$ and $\tilde{f}$

$$
\begin{aligned}
& \tilde{f}=\alpha \kappa \partial_{x} \rho \\
& \tilde{h}=\alpha \kappa \partial_{x}(\rho e)
\end{aligned}
$$

is known to be positive [22].

It remains to obtain a definition for the dissipative term $l$ used in the volume fraction equation. A way to achieve this, is to consider the volume fraction equation by itself and notice that it is a hyperbolic equation with the eigenvalue $u_{I}$ (indeed, $u_{I}$ is an eigenvalue, as well, of the 7-equation system). An entropy equation can be derived and used to prove the entropy minimum principle by properly choosing the dissipative term. The objective is to ensure positivity of the volume fraction and also uniqueness of the weak solution. Following the work of Guermond et al. in $[16,17]$ and by analogy to Burger's equation, it can be shown that a dissipative term ensuring positivity and uniqueness of the weak solution is of the form $l=\beta \partial_{x} \alpha$ where $\beta$ is a positive viscosity coefficient. 
All of the dissipative terms are now defined and are recalled here for convenience:

$$
\begin{aligned}
l & =\beta \partial_{x} \alpha \\
f & =\alpha \kappa \partial_{x} \rho+\rho l \\
g & =\alpha A \mu \rho \partial_{x} u \\
h & =\alpha \kappa \partial_{x}(\rho e)+u g-\frac{u^{2}}{2} f+\rho e l
\end{aligned}
$$

At this point, some remarks are in order:

1. The dissipative term $l$ requires the definition of a new viscosity coefficient $\beta$. It was shown that this viscosity coefficient is independent of the other viscosity coefficients $\mu$ and $\kappa$. Its definition should account for the eigenvalue associated with the void fraction equation $u_{I}$. In addition, an entropy residual can be determined by analogy to Burger's equation. It is noted, however, that the eigenvalue $u_{I}$ can be discontinuous since its definition involves the sign of the void fraction gradient, which makes the theory more challenging. For simplicity, we ignore this aspect of the theory in this report.

2. The dissipative term $f$ is a function of $l$. Thus, all of the other dissipative terms are also functions of $l$.

3. The partial derivatives $s_{e}$ and $s_{\rho}$ can be computed using the definition provided in (93), and are functions of the thermodynamic variables: pressure, temperature and density.

4. All of the dissipative terms, except the one in the volume fraction equation, are chosen to be proportional to the the void fraction $\alpha$ and the cross-sectional area $A$. For instance, $\alpha A \partial_{x} \rho$ is the flux of the dissipative term in the continuity equation through the area seen by the phase $\alpha A$. When one of the phases disappears, the dissipative terms must to go to zero for consistency. On the other hand, when $\alpha$ goes to one, the single-phase equation must be recovered.

It remains now to specify the coefficients $\kappa, \mu$, and $\beta$ in the artificial viscous terms of our balance equation system. In the current version of the method [21], $\kappa$ and $\mu$ are set for each phase as though that phase was a single phase only. Furthermore, they are set equal in each phase; that is $\kappa_{k}=\mu_{k}$ for $k=l i q$, vap. The current definition includes an upper bound coefficient that will give a first order viscosity, denoted with subscript max, and a coefficient that will give a high-order viscosity, denoted with subscript $e$. The first-order 
viscosity coefficients $\kappa_{\max , k}$ and $\mu_{\max , k}$ are proportional respectively to the largest local, phasic eigenvalue $\left|u_{k}\right|+c_{k}$ and their use is equivalent to an first-order upwind scheme

$$
\kappa_{\max , k}(x, t)=\mu_{\max , k}(x, t)=\frac{h}{2}\left(\left|u_{k}\right|+c_{k}\right)
$$

where $h$ is the grid size. Such schemes are known to be monotone, but overly dissipative. The higher-order viscosity coefficients $\kappa_{k, e}$ and $\mu_{k, e}$ are set proportional to the entropy production that is evaluated by computing the local entropy residual $D_{e, k}$ as

$$
D_{e, k}(x, t)=\frac{\partial s_{k}}{\partial t}+u_{k} \frac{\partial s_{k}}{\partial x}=\frac{s_{k, e}}{P_{k, e}} \underbrace{\left(\frac{d_{k} P_{k}}{d t}-c_{k}^{2} \frac{d_{k} \rho_{k}}{d t}\right)}_{\tilde{D}_{e, k}(x, t)}
$$

where $\frac{d_{k}}{d t}$ denotes the material- $k$ (or total- $k$ ) derivative, $P_{k, e}$ is the partial derivative of the phase- $k$ pressure $P_{k}$ with respect to the phase- $k$ internal energy $e_{k}$, and $s_{k, e}$ is the partial derivative of the phase- $k$ entropy with respect to the phase- $k$ internal energy $e_{k}$. The expression for the entropy residual has been written as a function of pressure and density. Because $D_{k, e}$ and $\tilde{D}_{k, e}$ are proportional to each other, the definition of the viscosity coefficients $\kappa_{k, e}$ and $\mu_{k, e}$ are written to depend upon $\tilde{D}_{e, k}$ as follows

$$
\kappa_{k, e}(x, t)=\mu_{k, e}(x, t)=h^{2} \frac{\max \left(\left|\tilde{D}_{e, k}(x, t)\right|, J\right)}{\left(1-M_{k}\right) \rho_{k} c_{k}^{2}+M_{k} \rho_{k}\left|u_{k}\right|^{2}}
$$

where $M_{k}$ is the phasic Mach number. The denominator has the same dimensions as pressure and is designed to ensure consistency when dealing with low Mach number flows. The jump $J$ is chosen to be proportional to the jump of the pressure and density gradients at the interfaces

$$
J_{i+1 / 2}=\left|u_{k}\right|_{i+1 / 2} \max \left(\llbracket \frac{\partial P_{k}}{\partial x} \rrbracket_{i+1 / 2}, c_{k}^{2} \llbracket \frac{\partial \rho_{k}}{\partial x} \rrbracket_{i+1 / 2}\right)
$$

where

$$
\llbracket q \rrbracket_{i+1 / 2} \equiv\left|q_{i}-q_{i+1}\right|
$$

denotes the jump in a given quantity $q$, and $i+1 / 2$ denotes the interface between cells $i$ and $i+1$. 
Lastly, the $\beta$ terms must be specified. By analogy to Burger's equation, an entropy residual $\mathcal{R}_{\alpha}$ can be derived from the volume fraction equation:

$$
\begin{aligned}
& \mathcal{R}_{\alpha}=\frac{\partial}{\partial t}(A \alpha)+u_{I} A \frac{\partial \alpha}{\partial x} \\
& \mathcal{R}_{\alpha}=\eta_{\alpha}\left[\frac{\partial}{\partial t}(A \alpha)+u_{I} A \frac{\partial \alpha}{\partial x}\right] \\
& \mathcal{R}_{\alpha}=A \frac{\partial \eta}{\partial t}+u_{I} A \frac{\partial \eta}{\partial x}
\end{aligned}
$$

where $\eta_{\alpha}$ is the derivative of an entropy function $\eta$ (of the volume fraction evolution equation) with respect to $\alpha[16,17,23]$, and $A$ is to be space-dependent only. The entropy residual $\mathcal{R}_{\alpha}$ will be peaked only in the presence of a shock in the volume fraction profile. By analogy to Burger's equation, we choose to define the viscosity coefficient $\beta$ proportional to the entropy residual $\mathcal{R}_{\alpha}$ as follows:

$$
\beta_{k}=h^{2} \max \left(\left|\mathcal{R}_{\alpha}\right|, J_{\alpha}\right)
$$

where $J_{\alpha}$ is defined as the jump of the volume fraction gradient based on the same nomenclature as (108). Notice that, unlike the definitions of $\kappa$ and $\mu$ in (107), a normalization parameter is not required for the definition of $\beta$ since $\alpha$ is non-dimensional.

Because the heat addition and friction are entropy producing physical phenomena and because those sub models are still evolving within the RELAP-7 development (as are other sub models, numerics, and coding), so too must the specific details of the entropy viscosity stabilization method. Here, only the fundamentals of the method in their current state have been presented.

\subsection{Examples}

Numerical results for the 7-equation two-phase model are presented in this section. The objective is to demonstrate that the entropy-viscosity method when applied to the 7-equation model yields the correct numerical solution. All of the numerical results were run with the Stiffened Gas equation of state described in (Section 3.2.5) for both the vapor and liquid phases. The computational domain is made of three pipes $(1 \mathrm{~m}, 4 \mathrm{~m}$ and $1 \mathrm{~m}$ ) linked by junction models. The respective number of elements used to spatially discretize the pipes are $(25,50,25)$. The area is constant and equal to $A=10^{-4} \mathrm{~m}^{2}$. Wall friction force and wall heat source are only allowed in the middle pipe so that the pressure, velocity 
and temperature profiles should be constant in the first and third pipes. Details regarding the type of boundary conditions used for the inlet and outlet boundaries are specified for each tests. Also, the pressure, temperature, velocity and viscosity coefficients profiles are shown. The system is initialized with a uniform pressure, $P a=6 \times 10^{6} \mathrm{~Pa}$, temperature, $T=507.41 \mathrm{~K}$, velocity, $u=2 . \mathrm{m} / \mathrm{s}$ and vapor volume fraction, $\alpha_{\text {cap }}=0.2$. All transients are integrated in time with second order backward differencing, BDF2.

\subsubsection{Constant wall heat source and wall friction}

The 7-equation model is run with a constant wall heat source $q_{w}$ and wall friction. The interfacial area $A_{\text {int }}$ coefficient is set to zero so that the two phases exchange neither mass nor energy. All of the heat transfer only occurs in the liquid phase. A static pressure boundary condition is used to model the outlet flow. We consider two types of inlet boundary condition: mass flow rate (MFR) and stagnation pressure (SGP) boundary. The corresponding boundary conditions are specified in Table 2 for each phase: We first show the numerical results obtained with the mass flow rate boundary conditions in Figure 3-Figure 6 In Figure 3, the liquid and vapor pressure are constant in the first and third pipes since they have no friction. In the middle pipe, the wall friction force makes the pressure decrease (the slope is almost constant because the flow is low Mach). The outlet pressure is equal to the static pressure specified for both phases. In Figure 4, the liquid temperature increases because of the constant wall heat source. Since the two phases are independent to each other, the vapor phase does not heat up and thus, the temperature profile remains constant. (The vapor temperature varies slightly because of the change in pressure due to the wall

friction. However, the variations are very small). The velocity profiles display some in-

\begin{tabular}{|c|c|c||c|}
\hline & \multicolumn{2}{|c||}{ Inlet } & Outlet \\
\hline MFR & $H\left(\mathrm{~J} \cdot \mathrm{kg}^{-1}\right)$ & $\rho u\left(k g \cdot \mathrm{m}^{2} \cdot \mathrm{s}^{-1}\right)$ & $P_{s}(\mathrm{~Pa})$ \\
\hline Liquid & $9.98 \times 10^{5}$ & 1617.42 & $6 \times 10^{6}$ \\
\hline Vapor & $2.78 \times 10^{6}$ & 52.88 & $6 \times 10^{6}$ \\
\hline SGP & $P_{0}(\mathrm{~Pa})$ & $T_{0}(\mathrm{~K})$ & $P_{s}(\mathrm{~Pa})$ \\
\hline Liquid & $6.001617 \times 10^{6}$ & 507.407 & $6 \times 10^{6}$ \\
\hline Vapor & $6.000052 \times 10^{6}$ & 507.406 & $6 \times 10^{6}$ \\
\hline
\end{tabular}

Table 2. Boundary conditions. 
teresting behavior in Figure 5 as well. In the first and third pipe, the velocity is constant for both phase since there is neither pressure gradient nor wall heat source. In the middle pipe, the liquid velocity increases mainly because of the wall heat source. The very small variation in the vapor velocity are due to the wall friction force. Lastly, the viscosity coefficients are plotted in Figure 6 on a logarithm scale. The high-order viscosity coefficients are multiple orders of magnitude smaller than the first-order viscosity coefficients, as expected. The liquid high-order viscosity coefficient is larger than that of the vapor phase because of the wall-heat source that will produce entropy in the middle pipe. Overall, the profiles are smooth and the numerical solutions match the boundary values: the entropyviscosity method seems to stabilize the scheme without altering the physical solution.

In Figure 7-Figure 10, numerical results for the 3-pipe system are shown when using a stagnation pressure boundary condition for the inlet boundary. The same conclusions as before can be drawn. It may be noticed that the vapor pressure and velocity profiles display some small instabilities that come from the software used for plotting.

We also investigated the impact of the first-order viscosity upon the numerical solution. The objective is to show the benefits of using a high-order stabilization method. When using the first-order viscosity coefficient instead of the high-order one, the system becomes overly dissipative and can affect the numerical solution. The 3-pipe system is run with the first-order viscosity and the steady-state profiles are compared against the ones obtained with the high-order viscosity shown in Figure 3-Figure 6. Even if a steady-state solution is obtained, the numerical solution is affected by the artificial dissipative terms as shown in Figure 11 for the liquid and vapor temperature profiles.

\subsubsection{Constant wall temperature and wall friction}

We still investigate the 3-pipe system described in Section 4.5.1 but with a constant wall temperature instead of a constant wall heat flux. With this assumption, the heat flux is no longer constant and is a function of the difference between the wall temperature and the fluid temperature. The inlet boundary is modeled by a mass flow rate boundary condition with the same parameters from Table 2. Numerical results are given in Figure 12Figure 15. The liquid and vapor pressure variations are very similar to what was observed in Section 4.5.1 since the pressure profile is mainly driven by the wall friction force. On the other hand, the liquid velocity profile is very different from what is obtained in Section 4.5.1: at steady-state, it can be shown that the velocity gradient is proportional to the wall heat flux in the low Mach asymptotic limit. In this pipe section, the wall heat flux is not constant but varies spatially, unlike in Section 4.5.1 where the wall heat flux was set 
constant.

\subsubsection{Mass and heat exchange terms, and wall friction}

We next allow mass and heat exchanges between the vapor and liquid phases. The interfacial area coefficient $A_{\text {int }}$, is set equal to 500. A 3-pipe system is still considered with wall friction but without wall heat source. A mass flow rate and a static pressure boundary conditions are used at the inlet and outlet boundaries, respectively (see Table 2). The code is run until steady state is reached. The numerical solutions are given in Figure 16-Figure 18.

Because of the relaxation terms, the two phases exchange mass and heat. The value of $A_{\text {int }}$ chosen is sufficiently large to keep the two phases in a quasi-equilibrium state. This is what is observed in the pressure profiles which are nearly the same for both phases. The velocities are very close as well and only differ in the middle pipe because of the wall friction force.

\subsubsection{1-D converging-diverging duct with relaxation terms}

A 1-D converging-diverging duct of length $1 \mathrm{~m}$ with the spatially dependent area $A(x)=$ $1+0.5 \cos (\pi x)$, is now considered. It is discretized spatially with 100 elements. The pressure and velocity relaxation terms are turned on and the interfacial area coefficient $A_{\text {int }}$ is set equal to 1700 . The system is expected to reach a steady-state solution. A stagnation pressure boundary condition is used at the inlet boundary by setting $P_{0}=1 \mathrm{MPa}$ and $T_{0}=453 \mathrm{~K}$. The static pressure $P_{s}=0.5 \mathrm{MPa}$ is set at the outlet boundary. The steadystate profiles of the pressure, velocity, volume fraction and viscosity coefficients are given in Figure 19-Figure 22. Because we set a large value for the interfacial area coefficient $A_{\text {int }}$, the two phases remain in a quasi-equilibrium: the pressure and velocity profiles are almost identical as shown in Figure 19 and Figure 20, respectively. In Figure 21, the liquid and vapor volume fraction profiles are given. The pressure relaxation term in the volume fraction equation makes the volume fraction vary. The challenge is to be able to stabilize the volume fraction with an adequate viscosity coefficient denoted by $\beta$. Its steady-state profile, along with the other viscosity coefficients for each phase, are plotted in Figure 22 on a logarithm scale. It is noticed that even if none of the viscosity coefficients saturate to the first-order viscosity, the numerical solution does not show any instabilities: the stabilization method is seen to efficiently stabilize the scheme whithout altering the physical solution. 


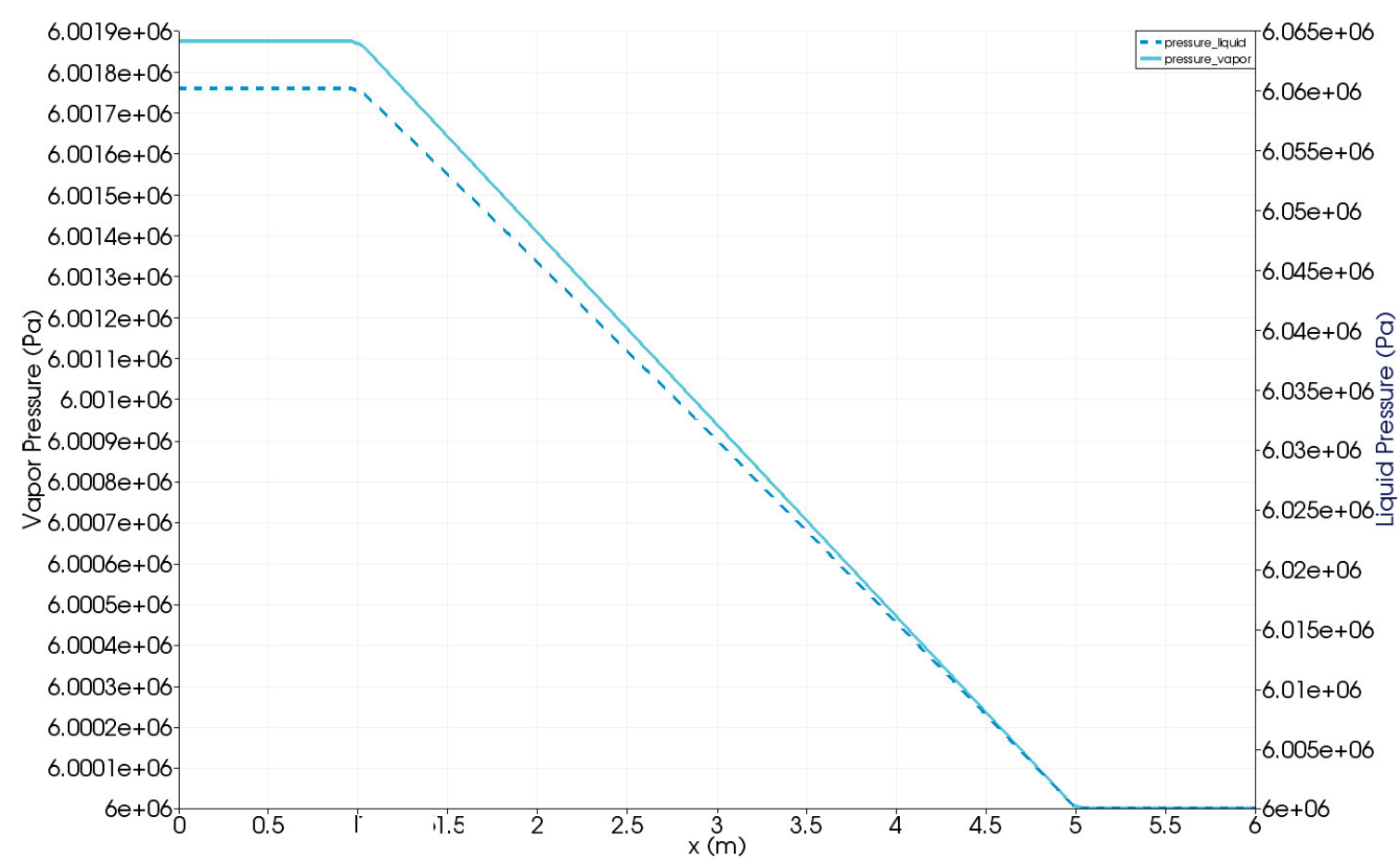

Figure 3. Steady-state pressure profile with constant wall-heat flux and mass flow rate boundary.

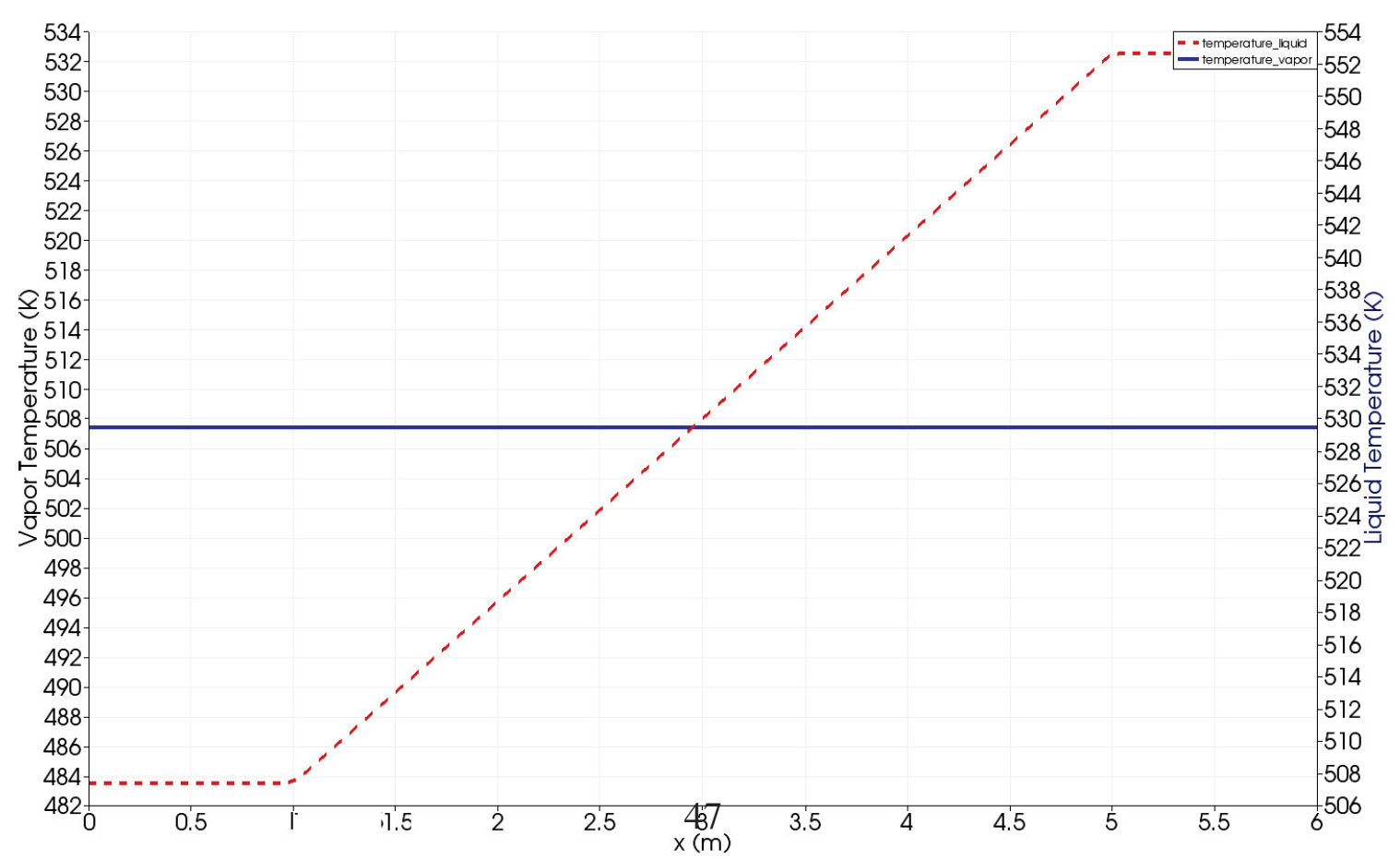

Figure 4. Steady-state temperature profile with constant wallheat flux and mass flow rate boundary. 


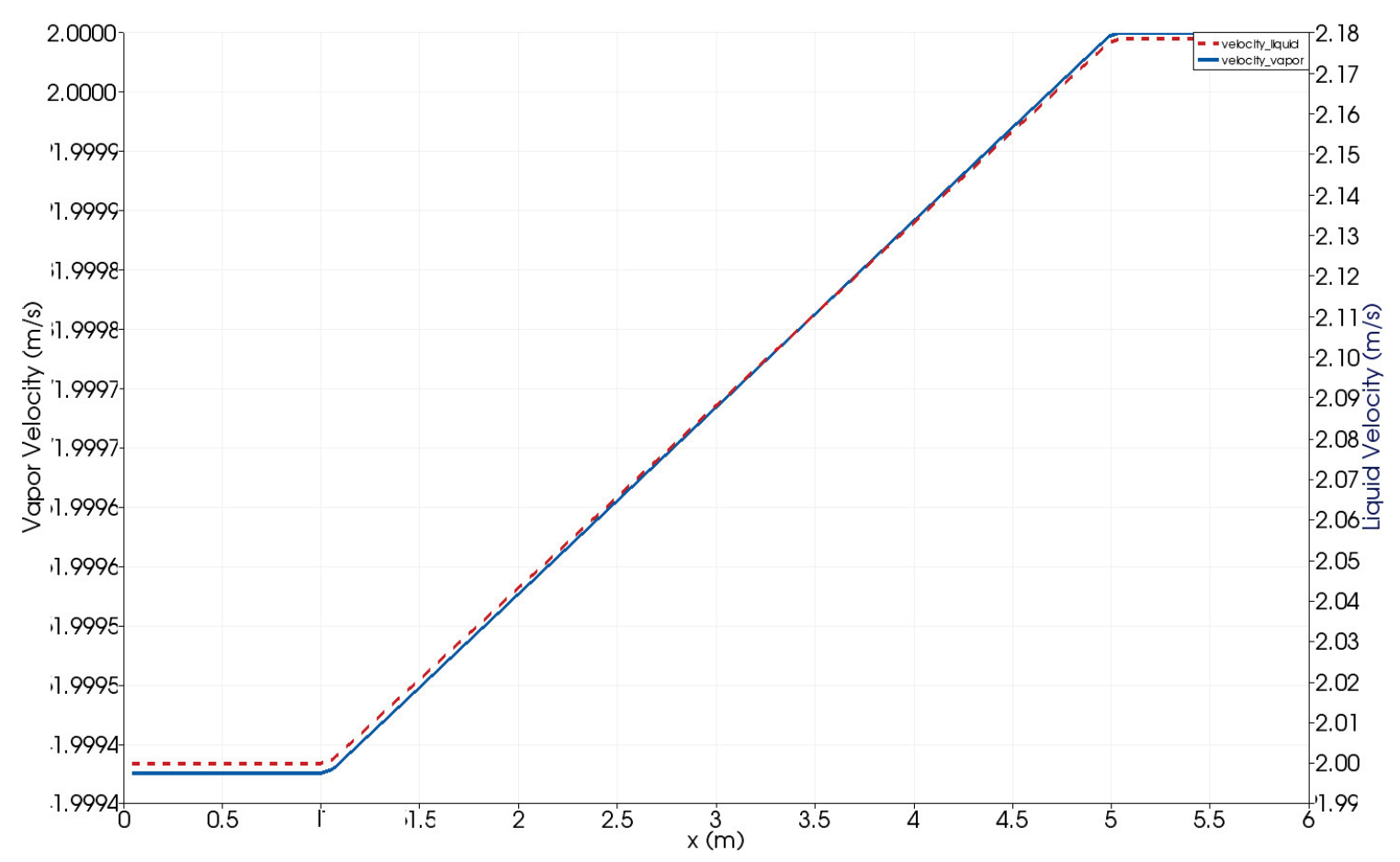

Figure 5. Steady-state velocity profile with constant wall-heat flux and mass flow rate boundary.

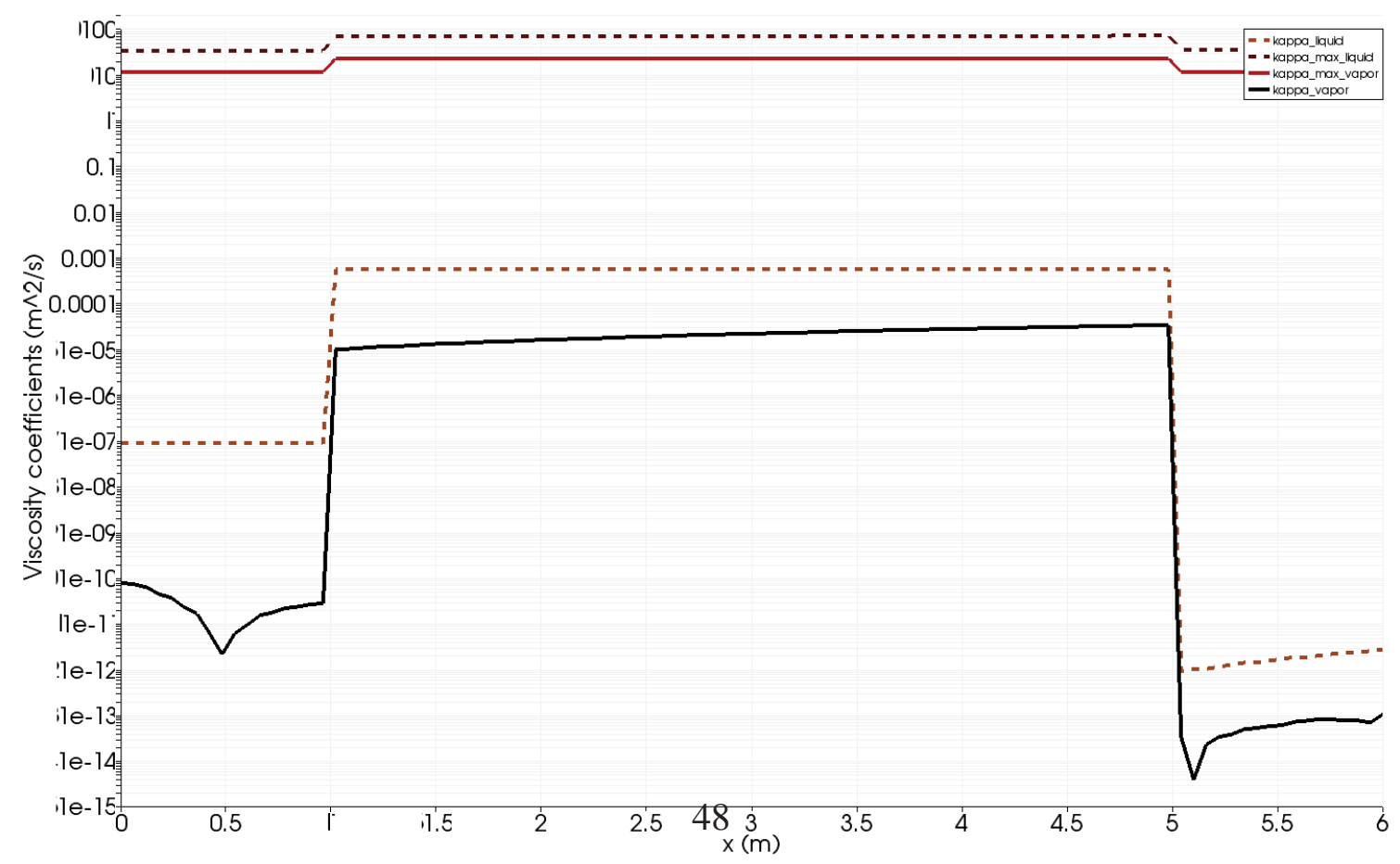

Figure 6. Steady-state viscosity coefficients profile with constant wall-heat flux and mass flow rate boundary. 


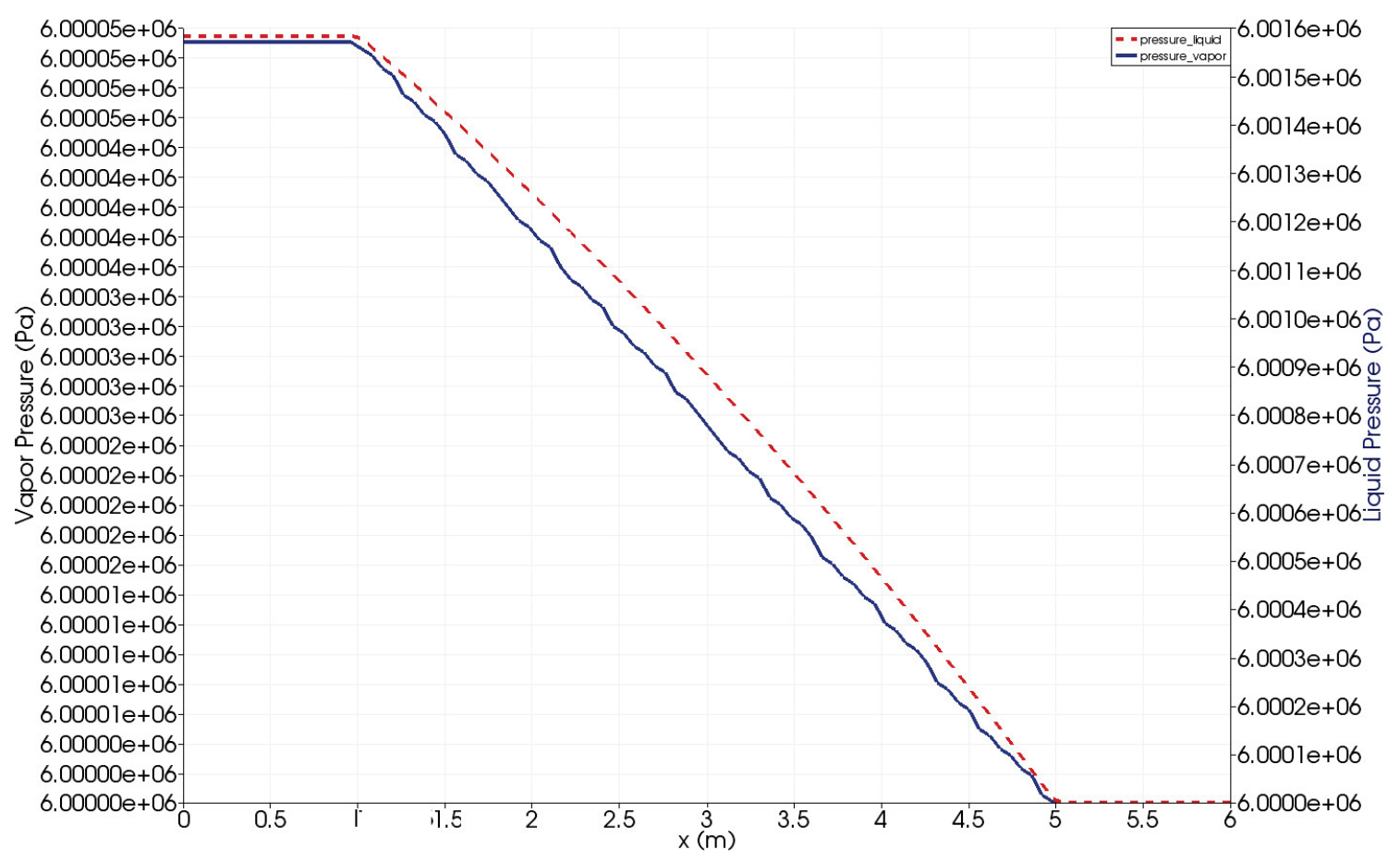

Figure 7. Steady-state pressure profile with constant wall-heat flux and stagnation boundary. 


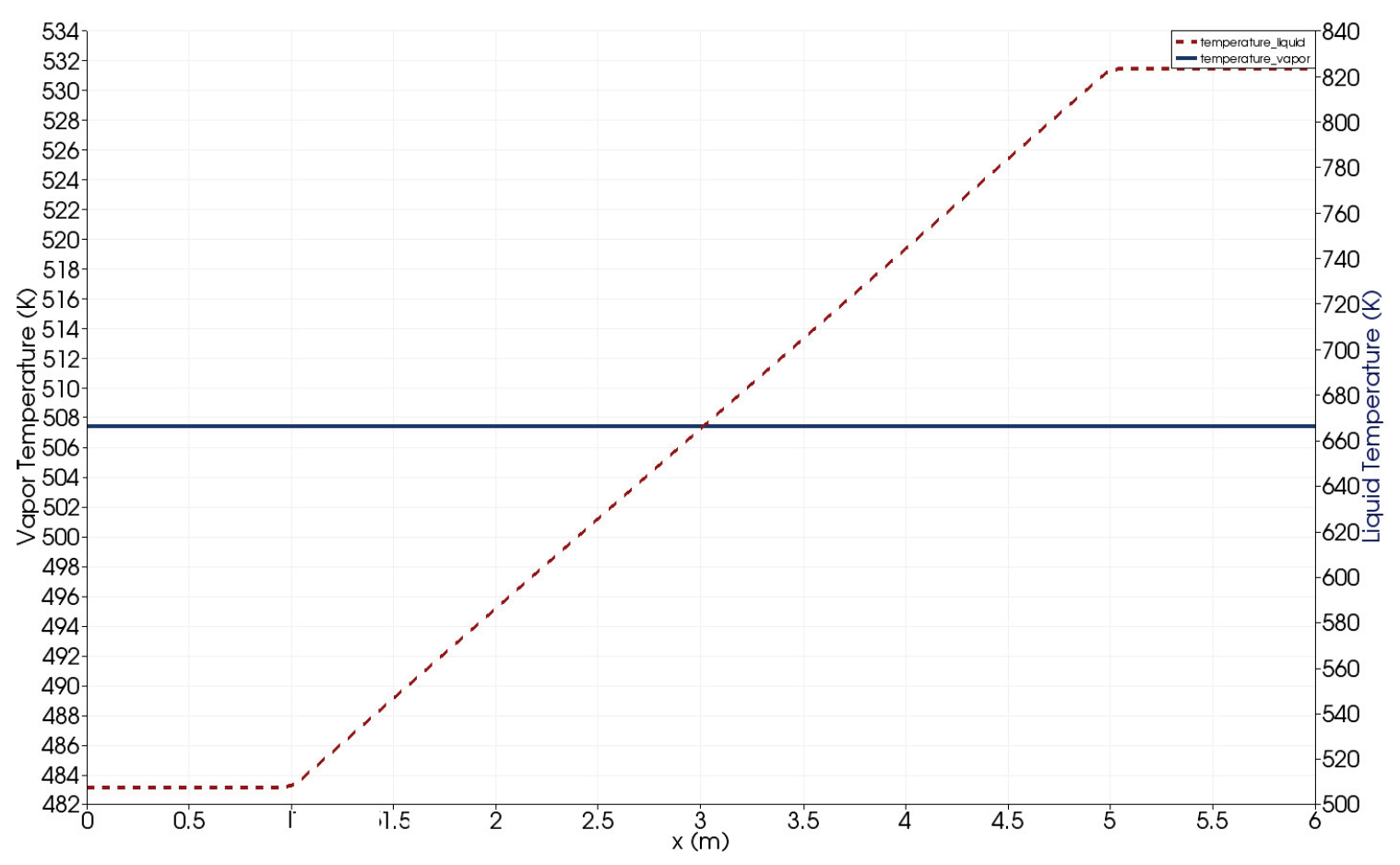

Figure 8. Steady-state temperature profile with constant wallheat flux and stagnation boundary. 


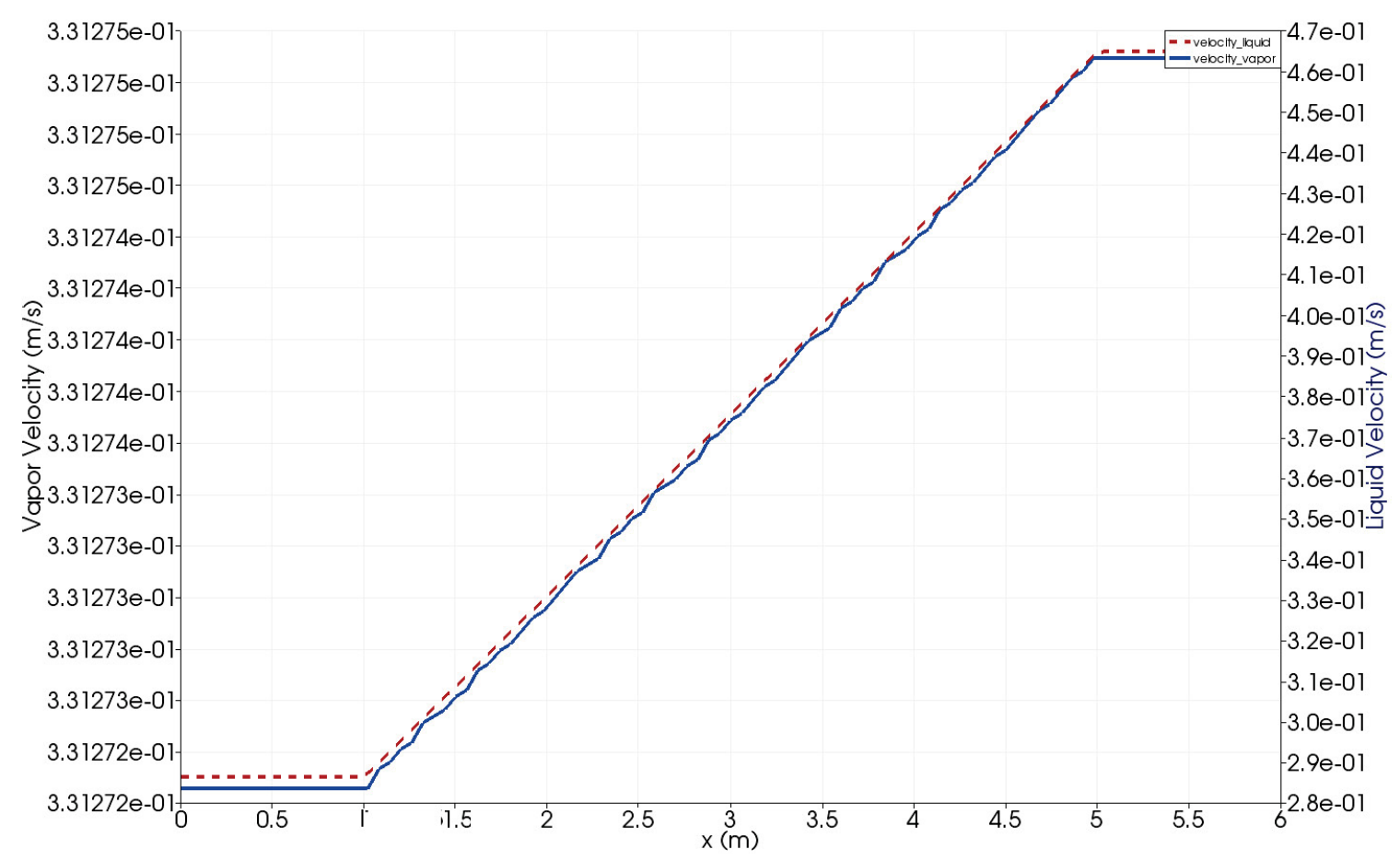

Figure 9. Steady-state velocity profile with constant wall-heat flux and stagnation boundary.

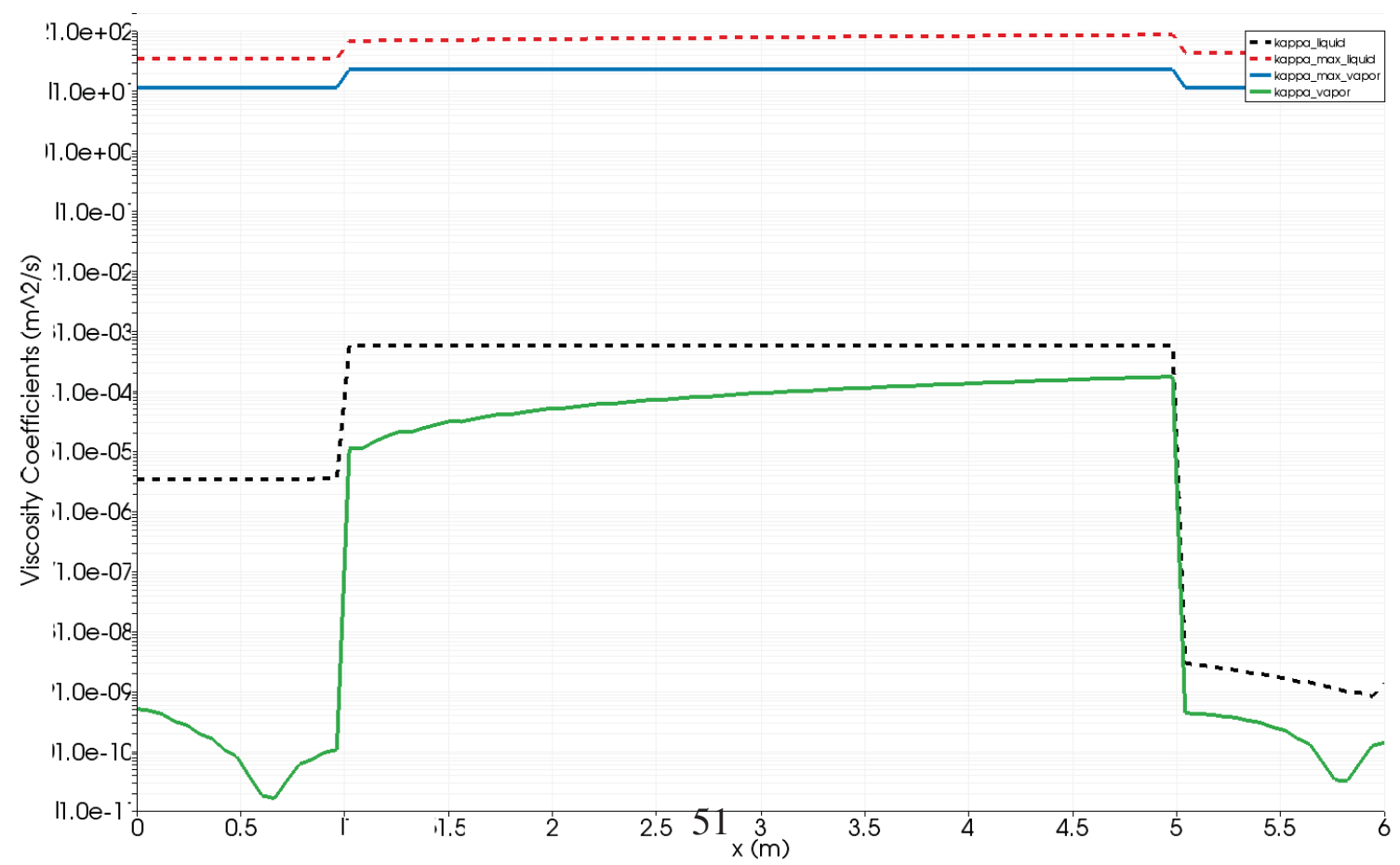

Figure 10. Steady-state viscosity coefficients profile with constant wall-heat flux and stagnation boundary. 


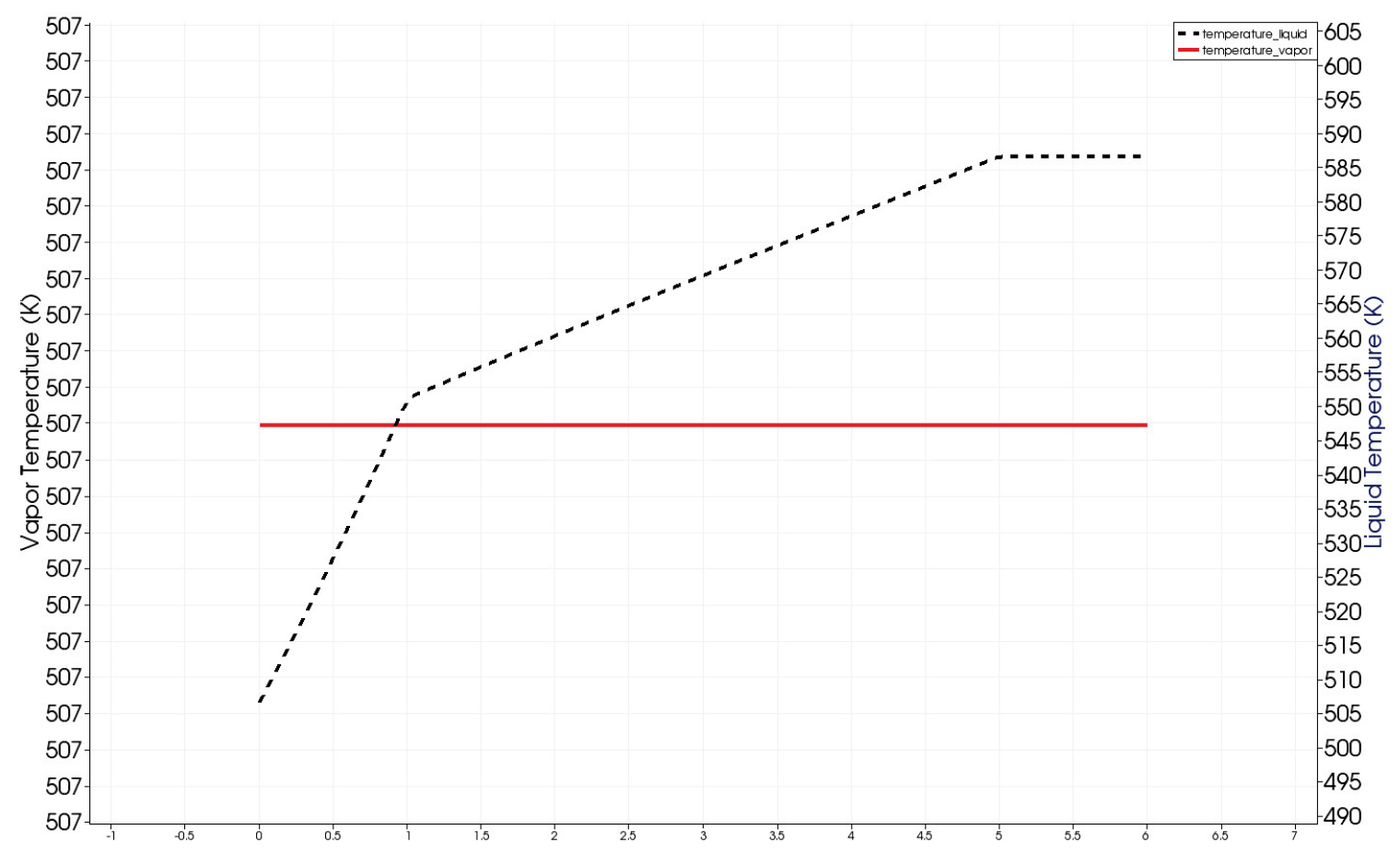

Figure 11. Steady-state temperature profile with constant wallheat transfer and first-order viscosity. 


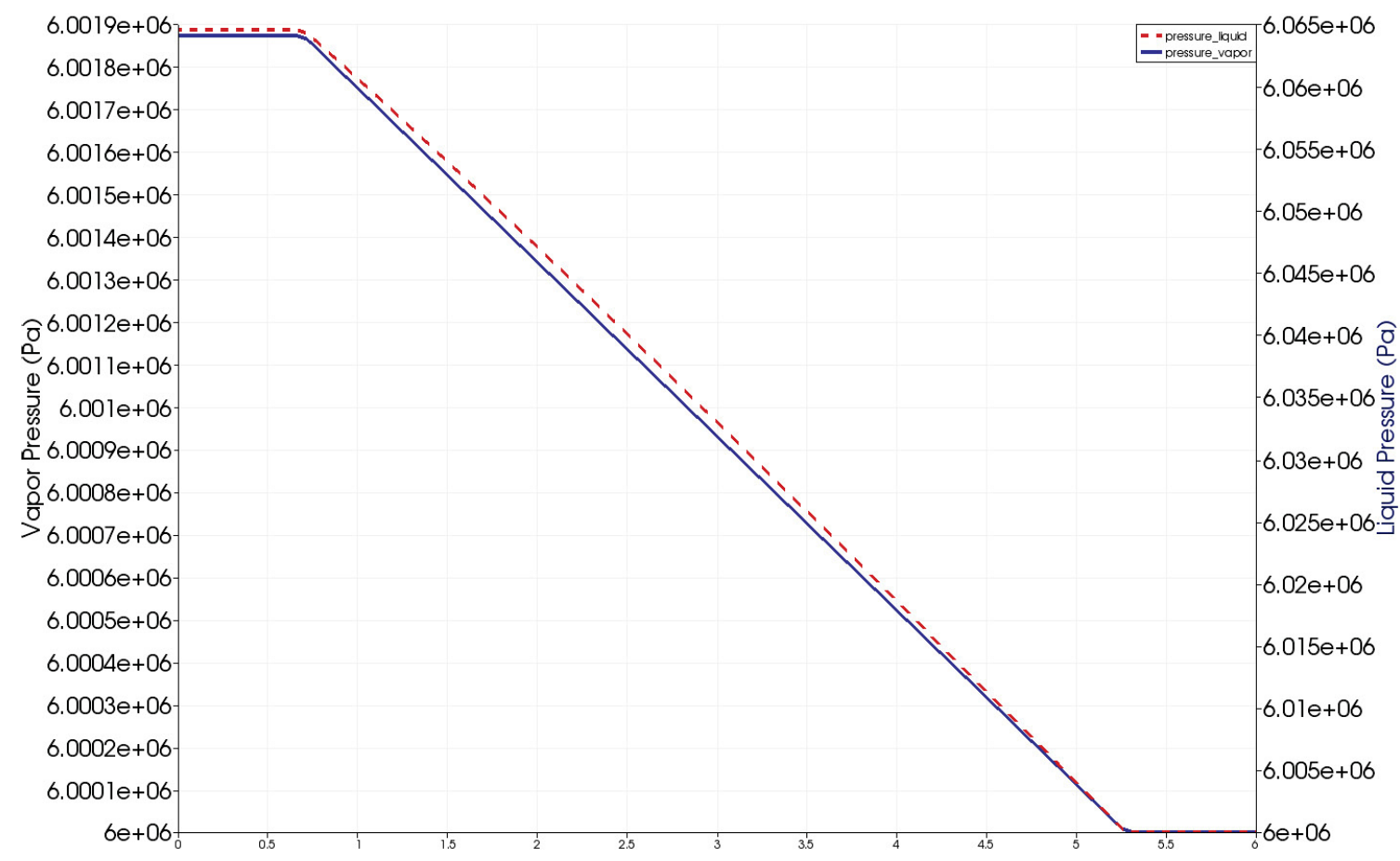

Figure 12. Steady-state pressure profile with constant wall temperature and mass flow rate boundary.

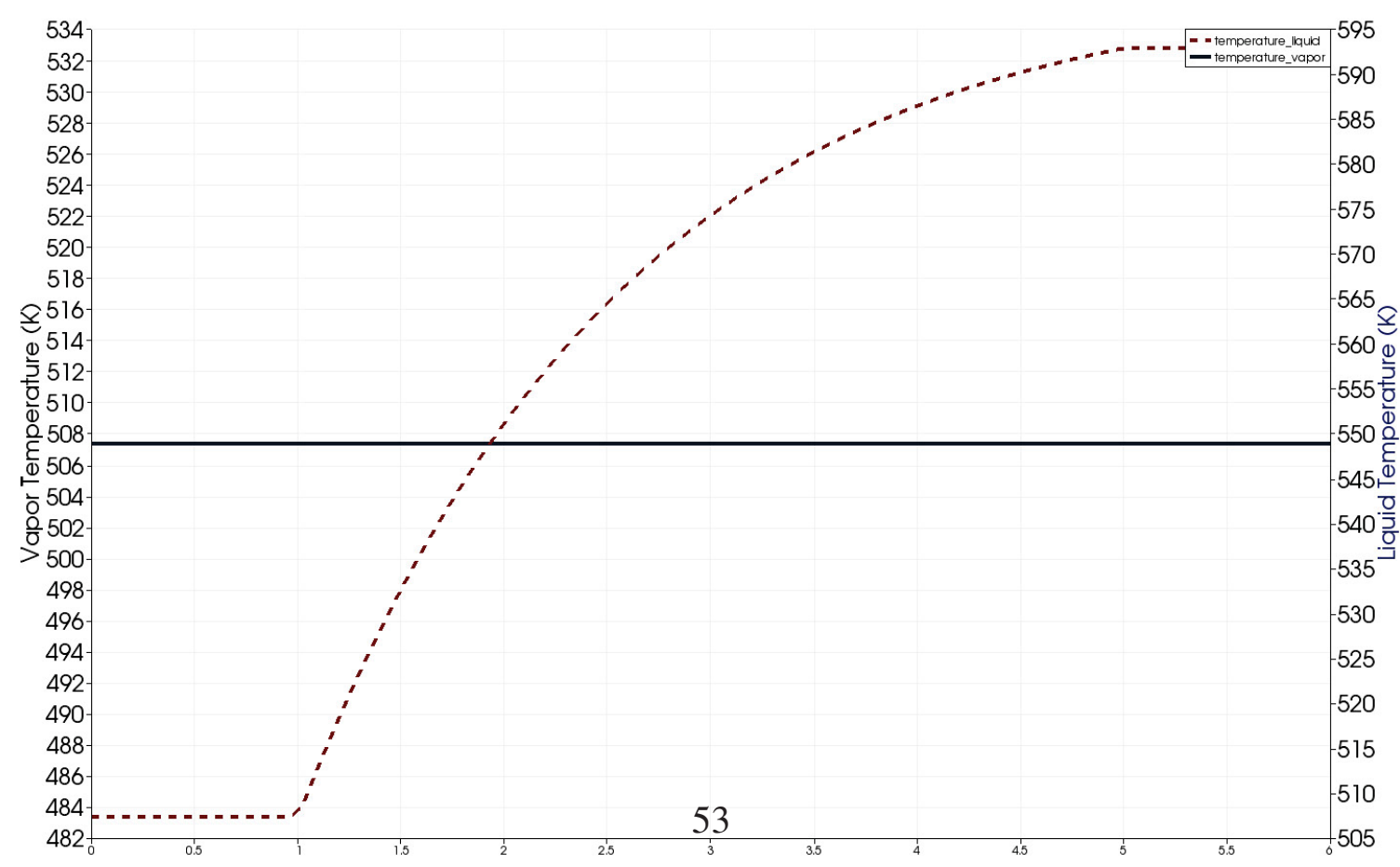

Figure 13. Steady-state temperature profile with constant wall temperature and mass flow rate boundary. 


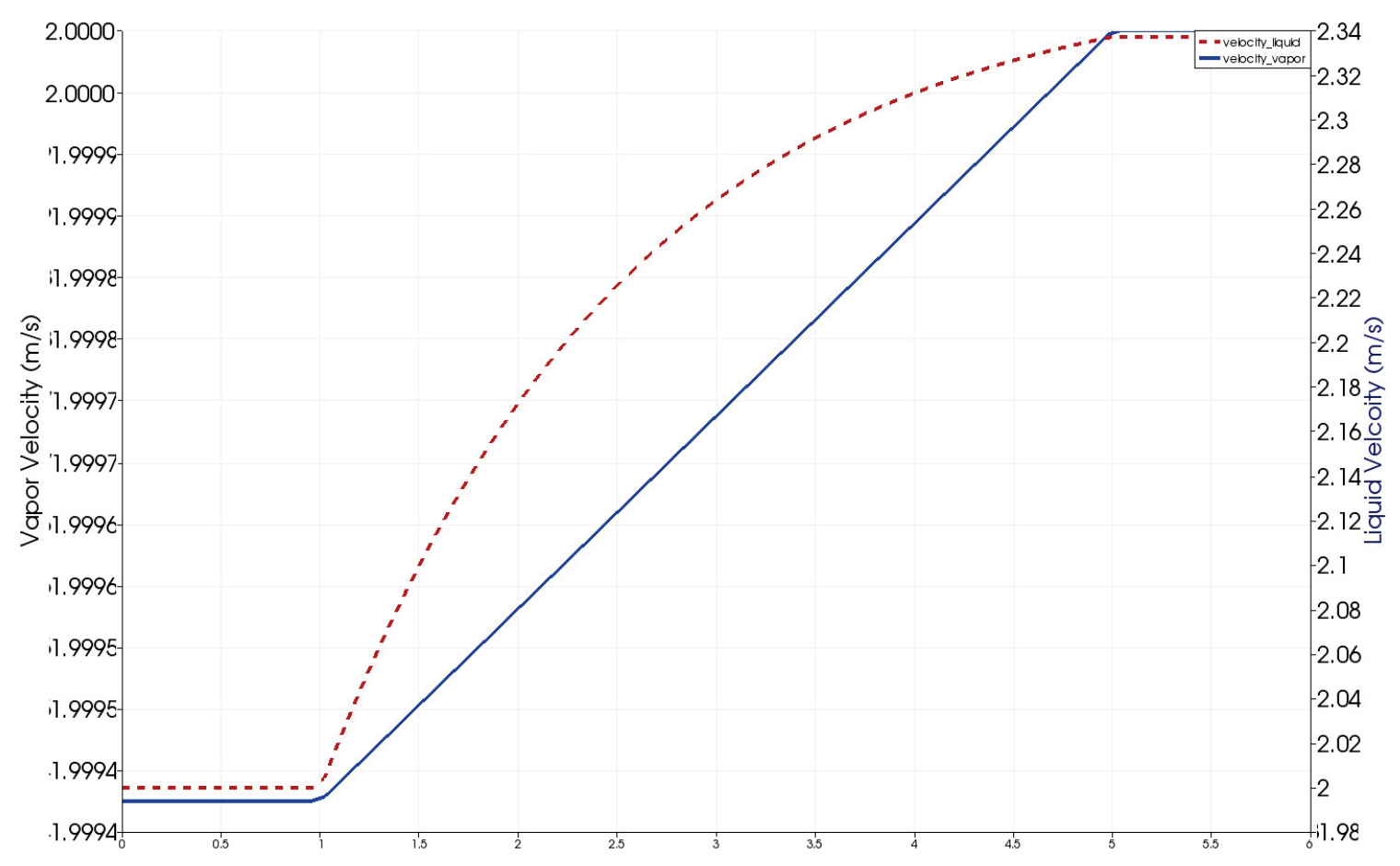

Figure 14. Steady-state velocity profile with constant wall temperature and mass flow rate boundary.

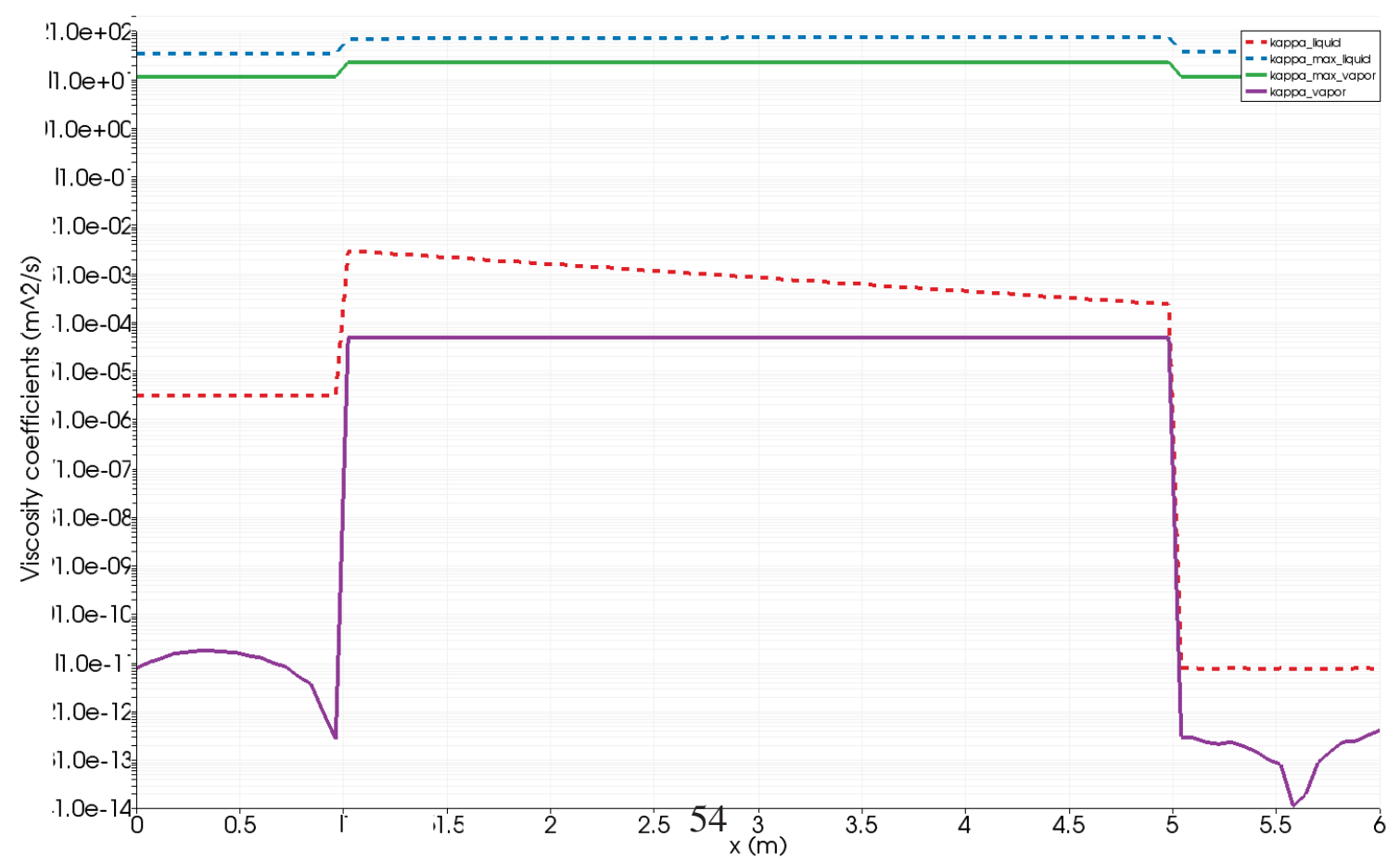

Figure 15. Steady-state viscosity coefficients profile with constant wall temperature and mass flow rate boundary. 


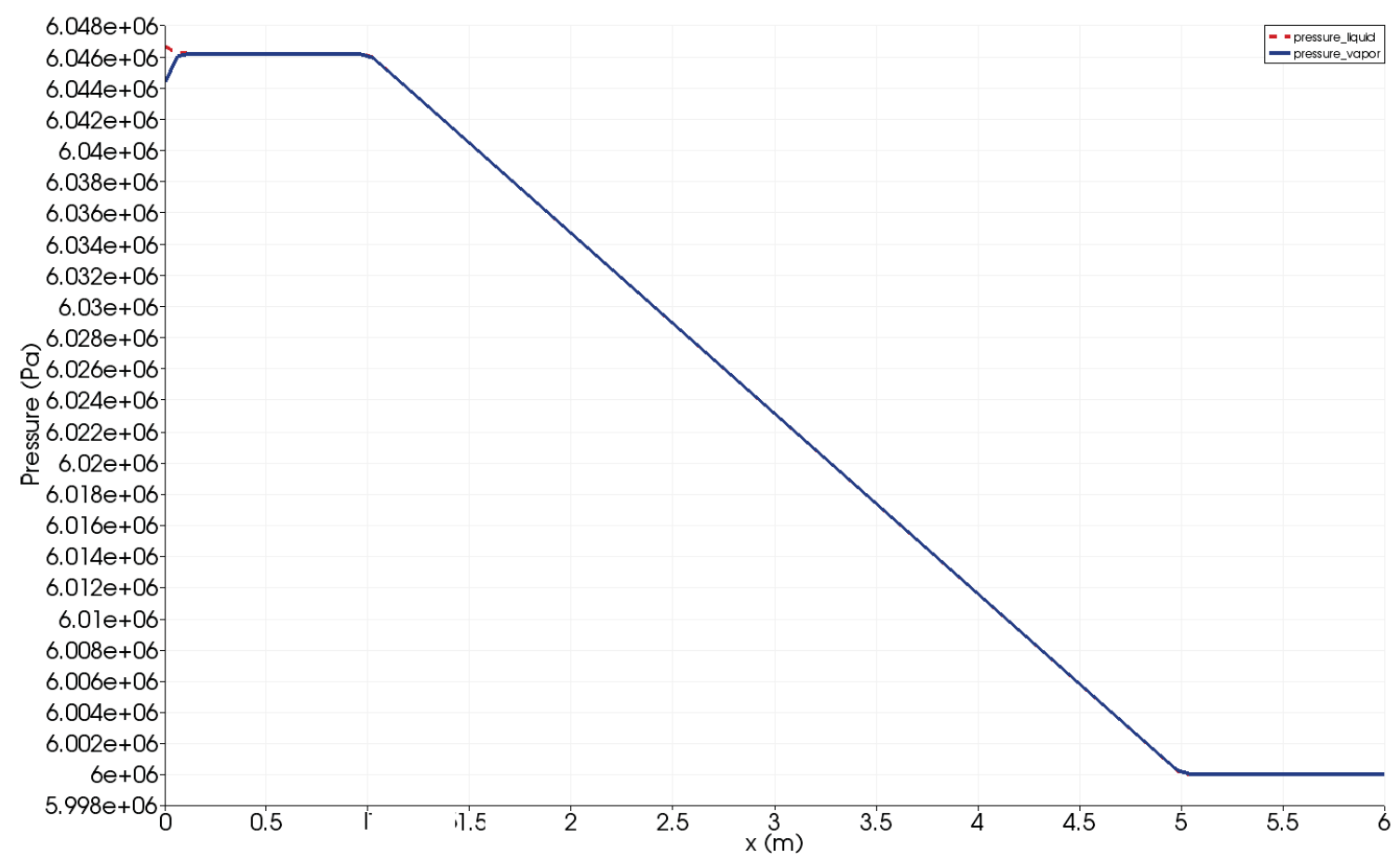

Figure 16. Steady-state pressure profile with friction and relaxation terms. 


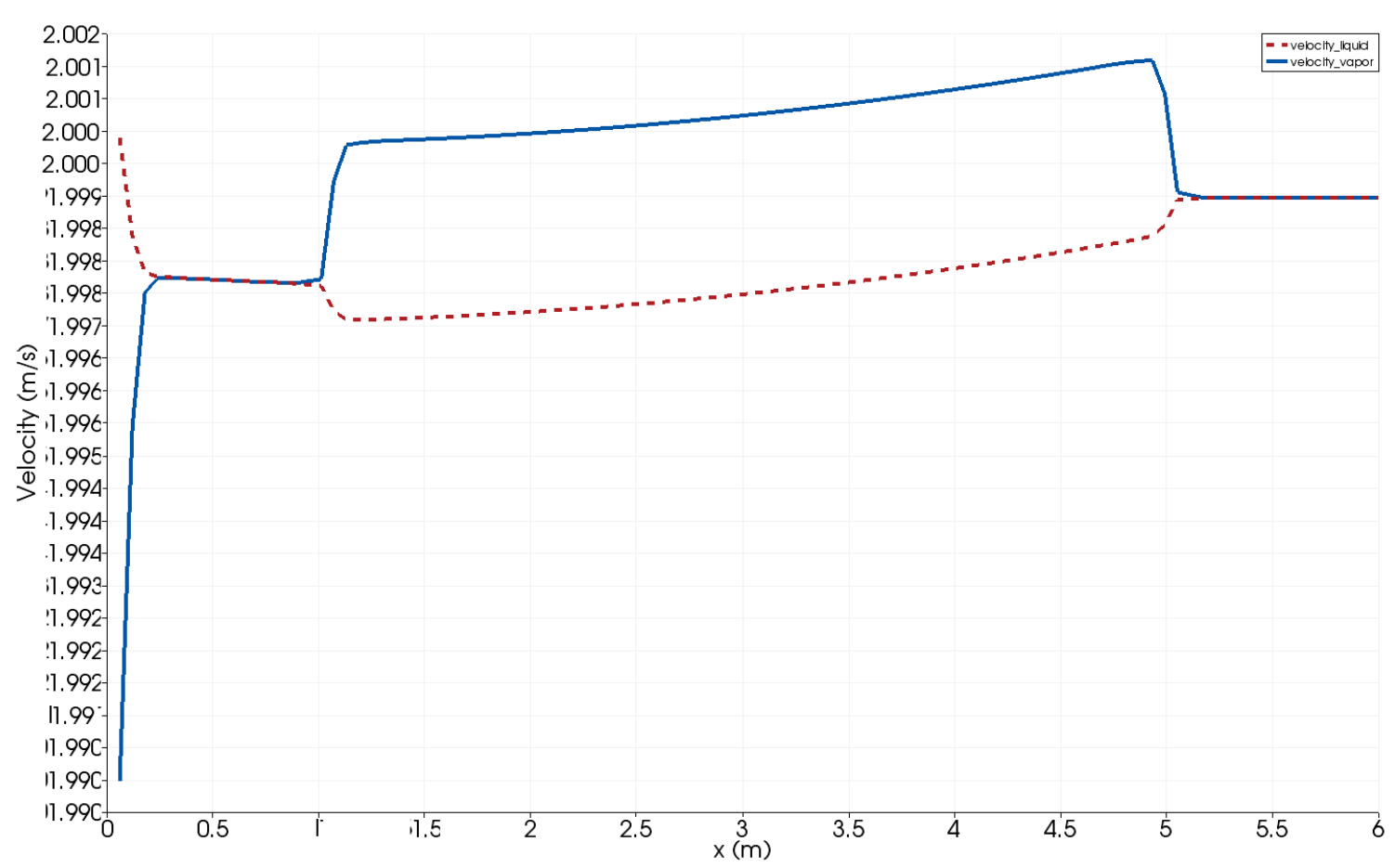

Figure 17. Steady-state velocity profile with friction and relaxation terms.

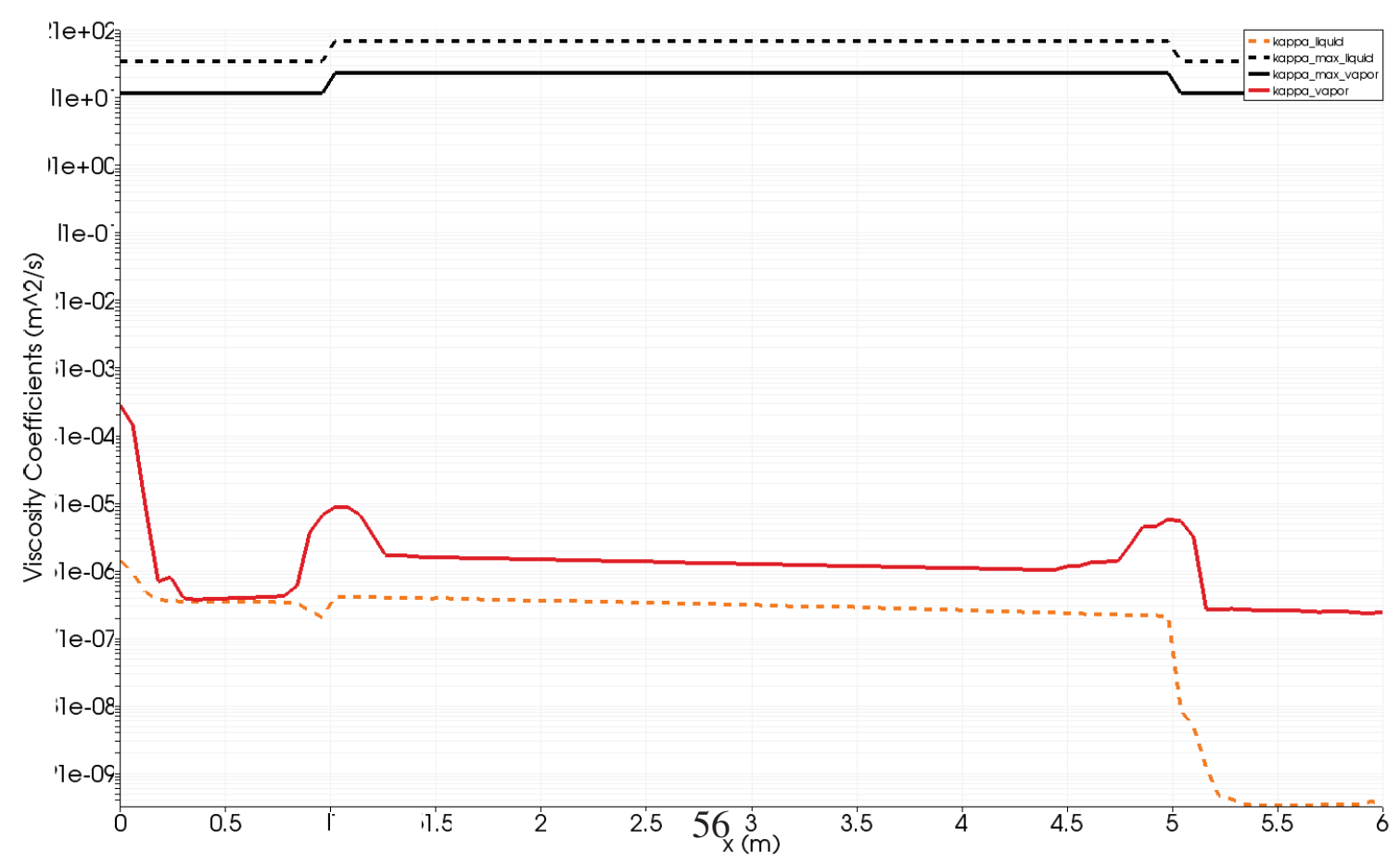

Figure 18. Steady-state viscosity coefficients profile with friction and relaxation terms. 


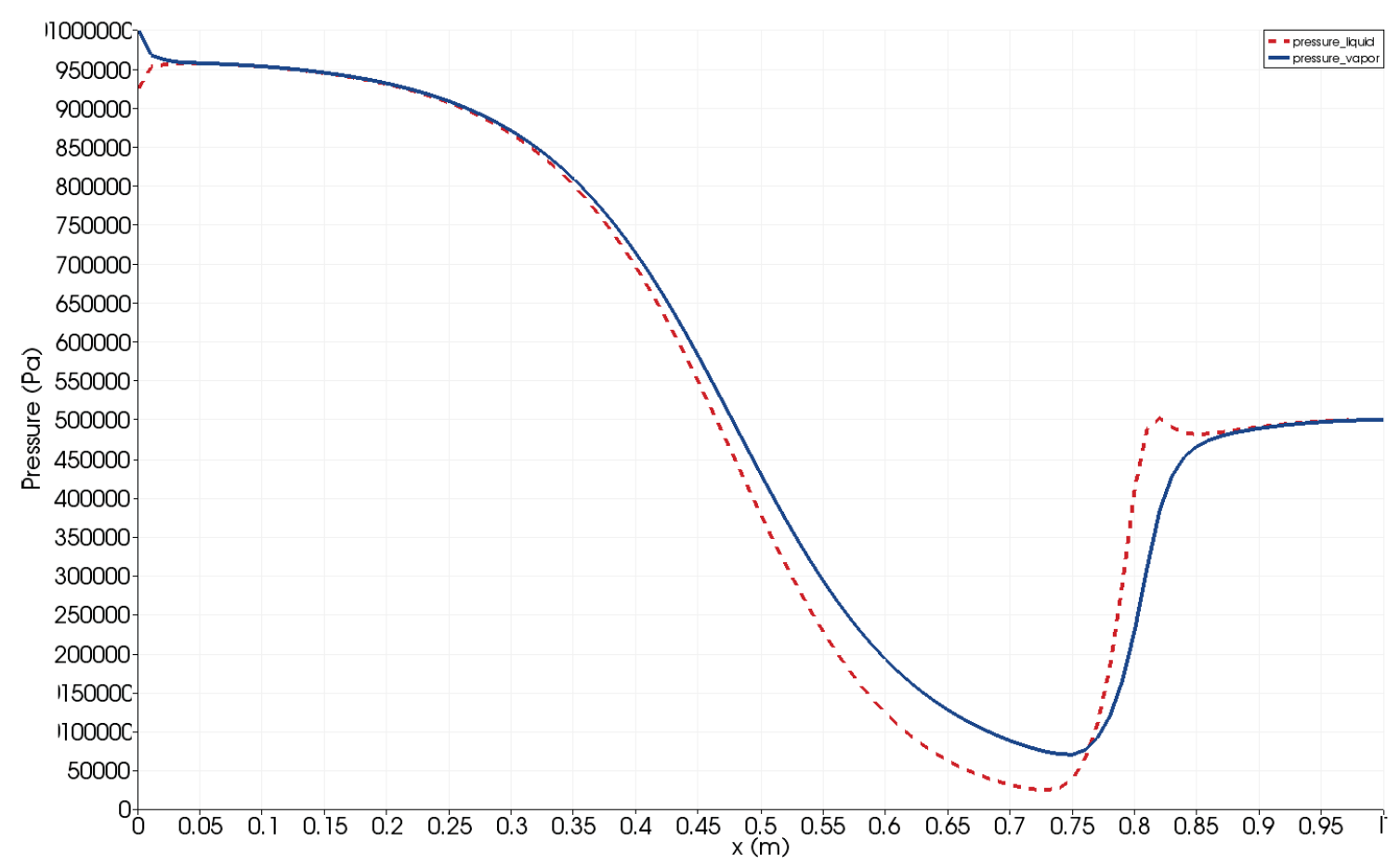

Figure 19. Steady-state pressure profiles in a convergingdiverging duct with pressure and velocity relaxation terms.

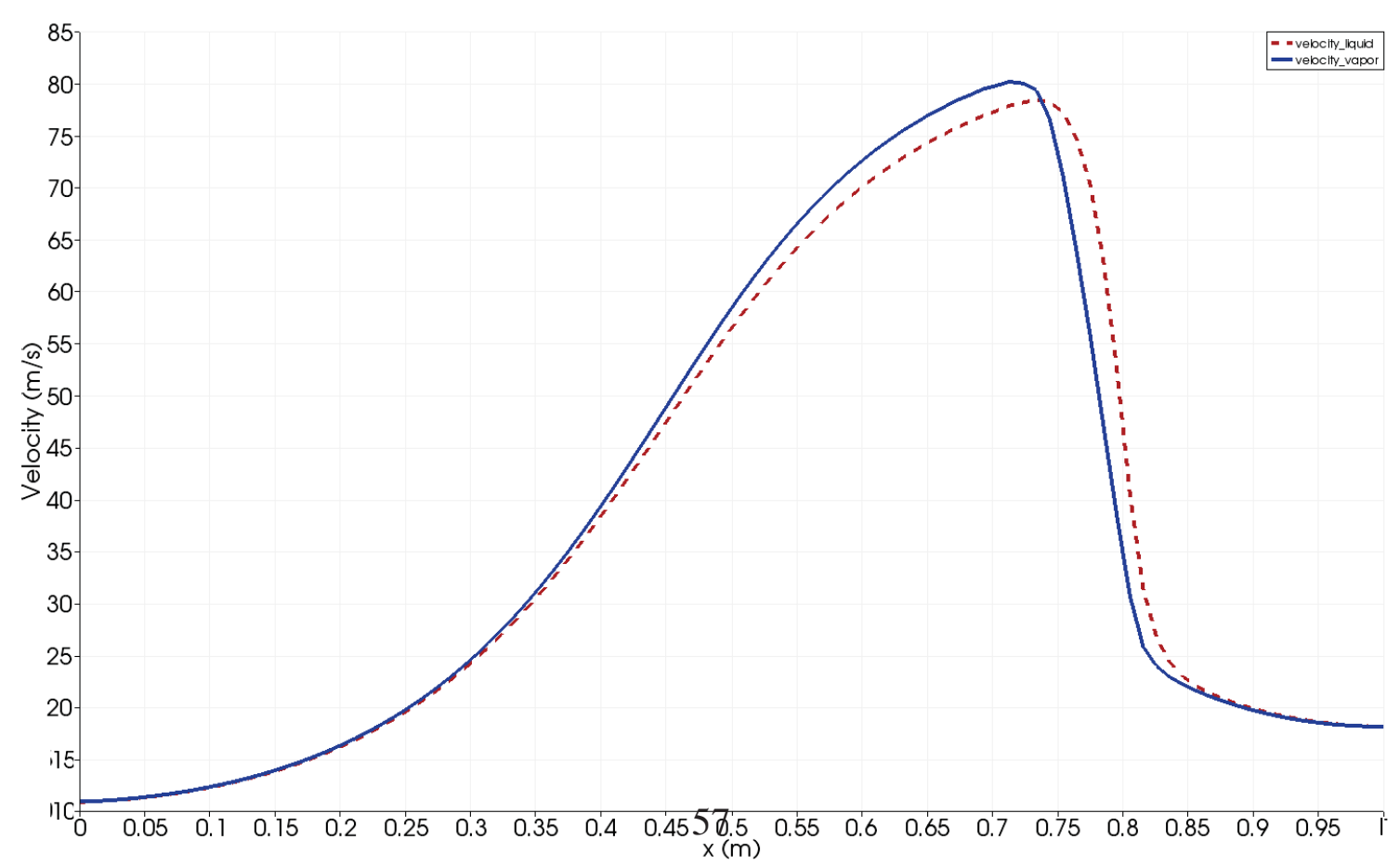

Figure 20. Steady-state velocity profiles in a convergingdiverging duct with pressure and velocity relaxation terms. 


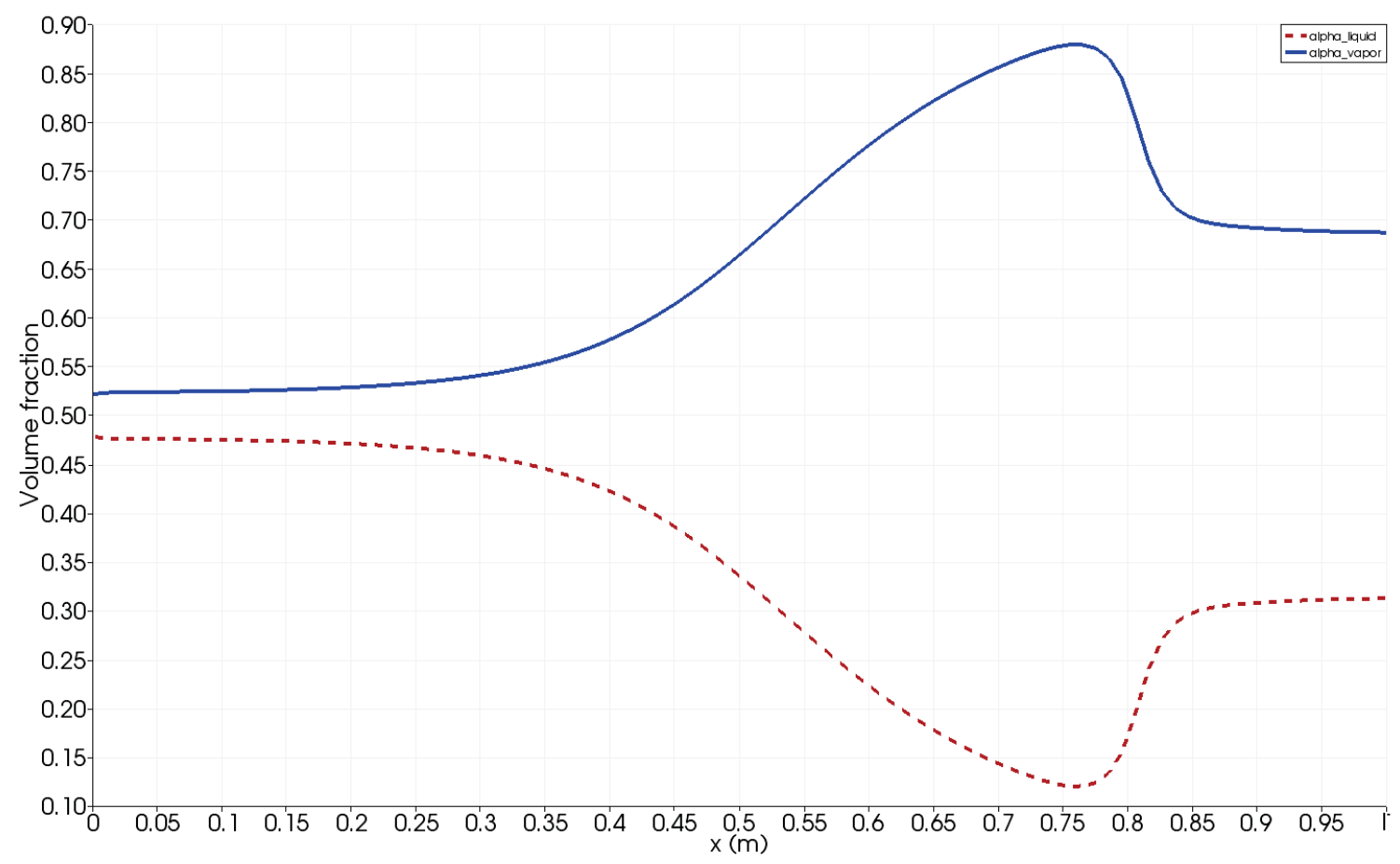

Figure 21. Steady-state volume fraction profiles in a convergingdiverging duct with pressure and velocity relaxation terms.

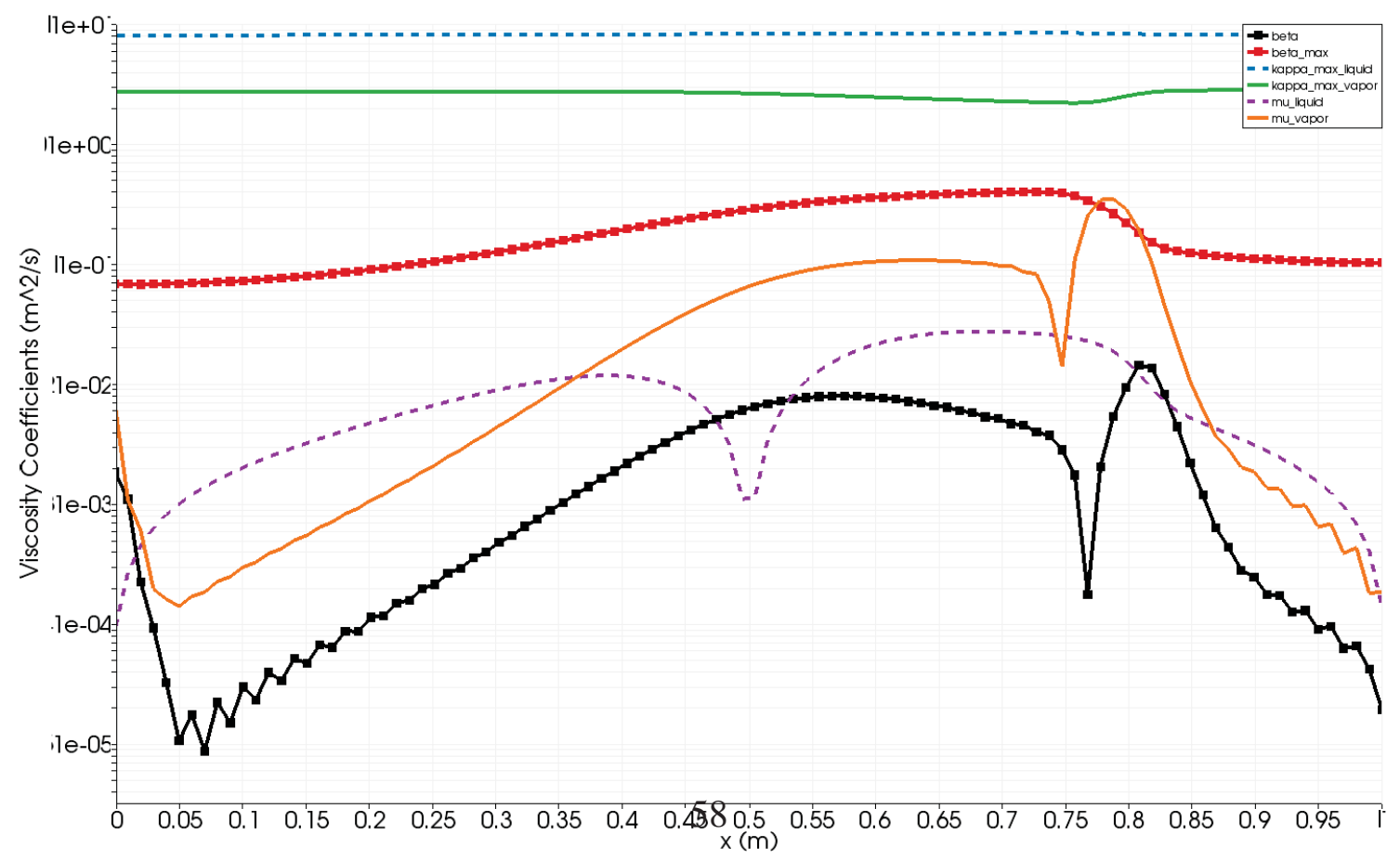

Figure 22. Steady-state viscosity coefficient profiles in a converging-diverging duct with pressure and velocity relaxation terms. 


\section{Summary}

In two-phase nuclear reactor flow applications, there can be a need to solve for complex flows wherein nearly incompressible or low Mach flow coexists with a sonic, or even supersonic, flow domain. This is due to the very large variation of the effective sound speeds. Even though normal flows may be in the nearly incompressible regime, compressible effects cannot be neglected because of heat sources. During blowdown and other possible transients, wherein flashing can occur, significant compressible vapor is present and choking may occur. Vapor collapse in low speed two-phase flows can produce significant pressure transient events due to compressibility of the fluids.

In this report, the descriptive hyperbolic equation systems for single- and two-phase flows used by RELAP-7, along with the numerical methods it employes, have been summarized. It was noted that accurate numerical solution of low Mach compressible flows can be problematic. To address this, details of an innovative numerical stabilization method recently incorporated into RELAP-7, the entropy viscosity method, has been presented. Examples have been given illustrating its application to some representative thermal hydraulic problems. For the problems illustrated the entropy viscosity method was seen to successfully and efficiently compute the low Mach compressible flows. 


\section{References}

[1] F. J. Moody, Introduction to Unsteady Thermofluid Mechanics. New Jersey: Wiley, 1990.

[2] O. L. Métayer, J. Massoni, and R. Saurel, "Elaborating equations of state of a liquid and its vapor for two-phase flow models," Int. J. Thermal Sciencies (in French), vol. 43, pp. 265-276, Mar. 2004. http://dx.doi.org/10.1016/ j.ijthermalsci.2003.09.002.

[3] R. A. Berry, J. W. Peterson, H. Zhang, R. C. Martineau, H. Zhao, L. Zou, and D. Andrs, "Relap-7 theory manual," Tech. Rep. INL/EXT-14-31366, Idaho National Laboratory, U. S. A., 2014.

[4] D. Gaston, C. Newman, G. Hansen, and D. Lebrun-Grandié, "MOOSE: A parallel computational framework for coupled systems of nonlinear equations," Nuclear Engineering and Design, vol. 239, pp. 1768-1778, Oct. 2009. http://dx.doi. org/10.1016/j.nucengdes.2009.05.021.

[5] R. A. Berry, "A logical progression of steps for implementation and testing of the 7equation, two-phase model into a computational framework," in Int. Conf. on Mathematics and Computational Methods Applied to Nuclear Science and Engineering, (LaGrange Park, IL), American Nuclear Society, 2013.

[6] R. A. Berry, R. Saurel, F. Petitpas, E. Daniel, O. LeMetayer, S. Gavrilyuk, N. Dovetta, and R. C. Martineau, "Progress in the development of compressible, multiphase flow modeling capability for nuclear reactor flow applications," Tech. Rep. INL/EXT-08-15002, Idaho National Laboratory, U. S. A., 2008.

[7] A. Chinnayya, E. Daniel, and R. Saurel, "Modelling detonation waves in heterogeneous energetic materials," J. of Computational Physics, vol. 196, pp. 490-538, 2004.

[8] R. A. Berry, R. Saurel, and O. LeMetayer, "The discrete equation method (DEM) for fully compressible, two-phase flows in ducts of spatially varying cross-section," Nuclear Engineering and Design, vol. 240, pp. 3797-3818, 2010.

[9] R. Saurel, F. Petitpas, and R. A. Berry, "Simple and efficient relaxation methods for interfaces separating compressible fluids, cavitating flows and shocks in multiphase mixtures," J. of Computational Physics, vol. 228, pp. 1678-1712, 2009. 
[10] J. M. Herrard and O. Hurisse, "A simple method to compute standard two-fluid models," Int. J. of Computational Fluid Dynamics, vol. 19, pp. 475-482, 2005.

[11] J. C. Butcher, Numerical Methods for Ordinary Differential Equations. New York: John Wiley \& Sons, 2003.

[12] U. M. Ascher and L. R. Petzold, Computer Methods for Ordinary Differential Equations and Differential-Algebraic Equations. Philadelphia: SIAM, 1998.

[13] D. A. Knoll and D. E. Keyes, "Jacobian-free Newton-Krylov methods: a survey of approaches and applications," Journal of Computational Physics, vol. 193, pp. 357397, Jan. 2004. http://dx.doi.org/10.1016/j.jcp.2003.08.010.

[14] R. Courant and K. O. Friedrichs, Supersonic Flow and Shock Waves. New York: Interscience, 1948.

[15] J. T. Oden and J. N. Reddy, An Introduction to the Mathematical Theory of Finite Elements. New York: Wiley \& Sons, 1976.

[16] J.-L. Guermond and R. Pasquetti, "Entropy viscosity method for higher-order approximations of conservation laws," Lecture Notes in Computational Science and Engineering, vol. 76, pp. 411-418, 2011.

[17] J.-L. Guermond, R. Pasquetti, and B. Popov, "Entropy viscosity method for nonlinear conservation laws,” J. of Computational Physics, vol. 230, pp. 4248-4267, 2011.

[18] M. O. Delchini, J. C. Ragusa, and R. A. Berry, "Entropy viscosity method applied to euler equations," in Int. Conf. on Mathematics and Computational Methods Applied to Nuclear Science and Engineering (M\&C2013), (LaGrange Park, IL), American Nuclear Society, 2013. Sun Valley, ID.

[19] V. Zingan, J.-L. Guermond, J. Morel, and B. Popov, "Implementation of the entropy viscosity method with the discontinuous galerkin method," Computational Methods in Applied Mechanics and Engineering, vol. 253, pp. 479-490, 2013.

[20] M. O. Delchini, "Extension of the entropy-based viscosity method to low-mach fluid flows and to seven-equation two-phase flows," tech. rep., Texas A\&M University, Nuclear Engineering Department, U. S. A., 2014. Ph.D. Dissertation, in preparation.

[21] M. O. Delchini, J. C. Ragusa, and R. A. Berry, "Extension of the entropy viscosity method to flows with friction forces and source terms," in Physor2014, (LaGrange Park, IL), American Nuclear Society, 2014. Kyoto, Japan. 
[22] J.-L. Guermond and B. Popov, "Viscous regularization of the euler equations and entropy principles," 2014. Under review.

[23] R. J. Leveque, Finite Volume Methods for Hyperbolic Problems. Cambridge, UK: Cambridge University Press, 2002. 


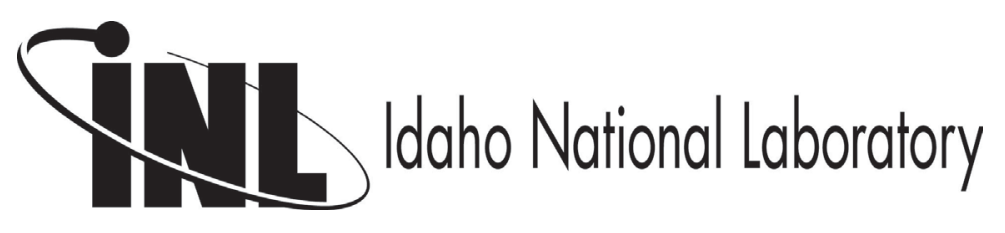

